\title{
Stimulation of Movement in a Quiescent, Hibernation-Like Form of Caenorhabditis elegans by Dopamine Signaling
}

\author{
Marta Maria Gaglia ${ }^{1,2}$ and Cynthia Kenyon ${ }^{1,2}$ \\ ${ }^{1}$ Neuroscience Program and Department of Biochemistry and Biophysics, and ${ }^{2}$ Hillblom Center for the Biology of Aging, University of California, San \\ Francisco, San Francisco, California 94158
}

One of the characteristics of animals in hibernation is reduced behavioral activity. The Caenorhabditis elegans dauer state is a hibernation-like state of diapause that displays a dramatic reduction in spontaneous locomotion. A similar dauer-like quiescent state is produced in adults by relatively strong mutations in the insulin/IGF-1 receptor homolog daf-2. In this study, we show that mutations affecting the neurotransmitter dopamine, which regulates voluntary movement in many organisms, can stimulate movement in dauers and dauer-like quiescent adults. Surprisingly, the movement of quiescent animals is stimulated by conditions that reduce dopamine signaling and also by conditions predicted to increase dopamine signaling. Reducing dopamine signaling is likely to stimulate movement by activating a foraging response also seen in nondauers after withdrawal of food. In contrast, the stimulation of movement by increased dopamine is much more pronounced in quiescent $d a f-2(-)$ dauer and dauer-like adult animals than in nondauaer animals. This altered response to dopamine is primarily attributable to activity of the FOXO (forkhead box 0) transcription factor DAF-16 in neurons. We suggest that dauers and dauer-like quiescent adults may have underlying changes in the dopamine system that enable them to respond differently to environmental stimulation.

\section{Introduction}

Adapting one's physiology to changing environmental conditions is essential for all living organisms. The Caenorhabditis elegans juvenile responds to conditions unfavorable for reproductive growth by altering its developmental trajectory and entering an alternative, hibernation-like larval stage called dauer (Cassada and Russell, 1975). Dauer larvae have unique morphological and behavioral features, including a marked reduction in movement, which are thought to allow them to survive harsh conditions. Because dauers can move when stimulated mechanically (Cassada and Russell, 1975), their quiescence appears to be a behavioral rather than a morphological adaptation. However, little is known about how their quiescence is influenced by neurotransmitter signaling.

We asked whether neurotransmitter systems that influence locomotion in C. elegans might also modulate dauer quiescence.

Received July 21, 2008; revised March 31, 2009; accepted April 6, 2009.

This work was supported by National Institutes of Health (NIH) Grant R01 AG11816 to C.K. Some nematode strains used in this work were provided by the Caenorhabditis Genetics Center, which is funded by the NIH National Center for Research Resources. We acknowledge the contributions to this work by the staff at the Neurochemistry Core, which is supported by National Institute of Child Health and Human Development Grant P3O HD15052 to the Vanderbilt Kennedy Center for Research on Human Development, and the Center for Molecular Neuroscience (Vanderbilt University Medical (enter, Nashville, TN). M.M.G. was supported by a Larry L. Hillblom Foundation Predoctoral Fellowship. We are grateful to A. Villu Maricq, Randy Blakely, Jim Rand, Bill Schafer, and Daniel Chase for sharing strains and constructs. We are very grateful to Miriam Goodman for allowing us to use the C. elegans tracker setup in her laboratory, and to Miriam Goodman, Daniel Ramot, and Brandon Johnson for help and discussion during the course of the tracker experiments. We thank all Kenyon Laboratory members for help and discussion, and in particular Robin Eisenhut and Ayumi Nakamura for technical help.

Correspondence should be addressed to Dr. Cynthia Kenyon, Department of Biochemistry and Biophysics, University of California, San Francisco, MC2200, Mission Bay Genentech Hall Room S312D, 600 16th Street, San Francisco, CA 94158-2517. E-mail: cynthia.kenyon@ucsf.edu.

D01:10.1523/JNEUROSCI.3429-08.2009

Copyright $\odot 2009$ Society for Neuroscience $\quad$ 0270-6474/09/297302-13\$15.00/0
We focused on the biogenic amine dopamine because dopamine regulates voluntary movement in many organisms. In C. elegans hermaphrodites, dopamine is produced by eight neurons (Sulston et al., 1975) and signals via at least four dopamine receptors, DOP-1 through DOP-4 (Suo et al., 2002, 2003; Chase et al., 2004; Sugiura et al., 2005). In addition, a presynaptic reuptake transporter, DAT-1, removes dopamine from the extracellular space to terminate signaling (Jayanthi et al., 1998; Nass et al., 2002).

In C. elegans, dopamine is best known for mediating the "basal slowing response" in which the animal's movement slows when it encounters food (Sawin et al., 2000). Dopamine is thought to be released in response to food and to act on motor neurons to modulate the animal's movement (Chase et al., 2004). Animals that cannot produce dopamine fail to slow in response to food (Sawin et al., 2000), and exogenous dopamine slows the movement of animals cultured without food (Schafer and Kenyon, 1995). The response to dopamine in this situation is complex, as the absence of specific dopamine receptors can have either positive or negative effects on movement (Chase et al., 2004).

In this study, we asked how conditions predicted to change the level of dopamine signaling influence the movement of dauers. Unexpectedly, we find that dauer movement can be stimulated either by increasing or decreasing the predicted level of dopamine signaling.

Dauers are developmentally arrested, prepubescent juveniles, but a dauer-like quiescent state can also be produced in the adult. Dauer formation is regulated by several signaling pathways, including the insulin/IGF-1 pathway (Riddle et al., 1981; Gottlieb and Ruvkun, 1994; Kimura et al., 1997; Riddle and Albert, 1997). Mutations that completely eliminate the function of DAF-2, the C. elegans insulin/IGF-1 receptor (InsR) (Kimura et al., 1997), 
cause constitutive dauer formation because of increased activity of the forkhead box O (FOXO) transcription factor DAF-16 (Riddle et al., 1981; Vowels and Thomas, 1992; Gottlieb and Ruvkun, 1994; Lin et al., 1997; Ogg et al., 1997; Riddle and Albert, 1997; Hu, 2007). Some slightly weaker daf-2/InsR mutations allow the animals to reach adulthood but produce a dauer-like quiescent behavior (Gems et al., 1998). We found that the movement of these quiescent adults was also stimulated by conditions predicted to either increase or decrease dopamine signaling.

Our findings suggest three conclusions. First, because neither reducing nor increasing the level of dopamine signaling produces dauer-like quiescence in normal animals, it is unlikely that dauer quiescence is caused simply by a change in dopamine-signaling strength. Second, because reducing food levels, like reducing dopamine levels, stimulates the movement of both dauer-like quiescent animals and nondauers, we infer that these quiescent animals respond to food withdrawal in a manner similar to nondauer animals. Third, because increasing dopamine signaling stimulates movement in daf-2(-) dauers and dauer-like adults, but not in nondauer animals, we infer that reductions in insulin/ IGF-1 signaling change the animals' response to dopamine. This altered response to dopamine is mediated in part by increased DAF-16/FOXO transcriptional activity in the nervous system.

\section{Materials and Methods}

\section{Strains}

Nematodes were raised under standard laboratory conditions on agar plates containing a lawn of Escherichia coli strain OP50, as described previously (Brenner, 1974). "Wild type" was the C. elegans strain N2. The mutant and transgenic strains used were as follows: CB1372 daf7(e1372), CF1041 daf-2(e1370), CF1085 daf-16(mu86); daf-2(e1370), CF1362 daf-16(mu86); daf-2(e1370); muEx142[rol-6(su1006)], CF1592 daf-2(e1370); muEx226[rol-6(su1006)], CF1794 daf-16(mu86); daf2(e1370); muIs109[Pdaf-16::gfp::daf-16; rol-6(su1006)], CF2005 daf16(mu86); daf-2(e1370); muIs120[Pges-1::gfp::daf-16; rol-6(su1006)], CF2093 daf-16(mu86); daf-2(e1370); muIs131 [Punc-119::gfp::daf-16; rol6(su1006)], CF2102 daf-16(mu86); daf-2(e1370); muIs126[Pmyo-3:: gfp::daf-16; rol-6(su1006)], CF2470 dat-1(ok157), CF2532 daf-2(e1370) dat-1(ok157), CF2593 cat-2(e1112), CF2625 daf-2(e1370); lin15(n765ts); akEx248[Pdat-1::gfp; lin-15(+)], CF2626 daf-2(e1370); lin-15(n765ts); akEx387[Pdat-1::gfp; Pdat-1::hICE; lin-15(+)], CF2646 daf-2(e1370); dop-3(vs106), CF2665 daf-2(e1370); dop-1(vs100) dop3(vs106), CF2666 daf-2(e1370); dop-1(vs100), CF2685 dat-1(ok157) daf-2(e1370); dop-1(vs100), CF2801 dat-1(ok157) daf-2(e1370); dop2(vs105), CF2802 dat-1(ok157) daf-2(e1370); dop-3(vs106), CF2803 dat-1(ok157) daf-2(e1370); dop-4(ok1321), CF2805 dop-2(vs105); dop-4(ok1321) dop-1(vs100) dop-3(vs106), CF2831 daf-2(e1370); dop2(vs105); dop-4(ok1321) dop-1(vs100) dop-3(vs106), CF3148 daf16(mu86); daf-2(e1370) dat-1(ok157), CF3248 daf-2(e1370); muIs176[Pdat-1::gfp; Pdat-1::hICE; Podr-1::rfp], CF3376 dat-1(ok157) daf-2(e1370); dop-2(vs105); dop-4(ok1321) dop-1(vs100) dop-3(vs106), CF3387 daf-2(e1370); dop-1(vs100); ljEx131[Pmec-7::dop-1; Punc122::gfp], CF3396 daf-16(mu86); daf-2(e1370); muEx536[Pacr-2:: gfp::daf-16; rol-6(su1006)], CF3397 daf-16(mu86); daf-2(e1370); muEx537[Pdat-1::gfp::daf-16; rol-6(su1006)], CF3399 daf-16(mu86); daf-2(e1370); muEx539[Posm-5::gfp::daf-16; rol-6(su1006)], CF3401 daf16(mu86); daf-2(e1370); muEx541[Pnmr-1::gfp::daf-16; rol-6(su1006)], CF3415 daf-16(mu86); daf-2(e1370); muEx545[Pglr-5::gfp::daf-16; rol6(su1006)], CF3417 daf-16(mu86); daf-2(e1370); muEx547[Punc-47:: gfp::daf-16; rol-6(su1006)], CF3420 daf-2(e1370); dop-1(vs100); muEx532[Pacr-2::dop-1; Podr-1::cfp], CF3421 daf-2(e1370); dop3(vs106); muEx534[Pacr-2::dop-3; Podr-1::cfp], JT195 daf-11(sa195). All mutations were backcrossed at least three times to our laboratory N2 strain, except the mutations in CB1372 and JT195.

Transgenic strains and constructs

Transgenic strains were created using standard methods (Mello and Fire, 1995).
pTH5 (Pdat-1::hICE) and pTH6 (Pdat-1::gfp) were kind gifts from A. Villu Maricq (University of Utah, Salt Lake City, UT) (Hills et al., 2004). They were injected into wild-type (N2) adults at a concentration of 10 ng/ $\mu$ l each, together with a Podr-1::rfp marker ( $40 \mathrm{ng} / \mu \mathrm{l})$. The transgenes were integrated using UV exposure to create $m u I s 176$, and the integrated lines were backcrossed six times to wild type (N2).

Pacr-2::dop-1 and Pacr-2::dop-3 were kind gifts from Daniel Chase (University of Massachusetts Amherst, Amherst, MA) (Chase et al., 2004). They were injected at $25 \mathrm{ng} / \mu \mathrm{l}$ into $d o p-1$ (vs100) and dop-3(vs106) animals, respectively, together with Podr-1::cfp (50 ng/ $\mu \mathrm{l})$ as a coinjection marker, to create muEx532 and muEx534.

Neuron-specific GFP::DAF-16 rescue constructs were generated from the same original construct used by Libina et al. (2003) to generate tissuespecific rescue constructs. A promoterless GFP::DAF16cDNA fusion construct (pCF105) was altered to insert a multiple cloning site (creating pCF231). Promoters were amplified with specific primers (sequence available on request) flanked with restriction sites, using Pfu Ultra High Fidelity (Stratagene). The lengths of the promoters were as follows: acr-2 (acetylcholinergic motor neurons): 3198 bp [based on Chase et al. (2004)]; dat-1 (dopamine-producing neurons): 1400 bp [based on Hills et al. (2004)]; glr-5 (interneurons): 1611 bp [based on Lee and Ashrafi (2008)]; $n m r-1$ (command interneurons): 4471 bp [based on Brockie et al. (2001)]; osm-5 (sensory neurons): 300 bp [based on Haycraft et al. (2001)]; unc-47 (GABAergic motor neurons): 262 bp [based on Chase et al. (2004)]. All promoters included sequences up to (but not including) the ATG start site. All constructs were injected into daf-16(mu86); daf2(e1370) double-mutant animals at $50 \mathrm{ng} / \mu \mathrm{l}$, and pRF4 [rol-6(su1009)] (Mello and Fire, 1995) was used as a coinjection marker (100 ng/ $\mu \mathrm{l}$ ). The expression pattern of each strain tested was verified. In all cases, it matched reported expression patterns. Green fluorescent protein (GFP) expression driven by Pacr-2 and Punc-47 was only clearly detected at larval stages.

\section{Movement assays}

Wild-type animals versus daf-2 mutant dauers. Wild-type (N2) and daf2(e1370) mutant animals were grown from eggs at $25^{\circ} \mathrm{C}$. Wild-type L1/L2 larvae were scored the day after moving the eggs to $25^{\circ} \mathrm{C}$ and wild-type L3/L4 the second day after transferring the eggs. Wild-type adults and daf-2(e1370) dauers were scored $3 \mathrm{~d}$ after transferring the eggs. All populations were scored at least three times.

Visual assay: mutant dauers. Movement of dauers was scored visually using a dissecting microscope. Dauers of strains carrying daf-2(e1370), daf-7(e1372), and daf-11(sa195) mutations were obtained by growing animals from eggs at $25^{\circ} \mathrm{C}$ for $3 \mathrm{~d}$ on plates containing food (E. coli OP50). Dauers were scored as moving if they moved at least one body bend forward or backward. A minimum of 30 animals per strain was scored in each repeat of the experiment. Experiments represented in Figures $1 B, 2 B, 6 D$, and $7 C$ were part of a set of experiments done in parallel, in which each strain was scored at least 11 times. Other dauer motility assays were repeated at least five times. The experiment in Figure $2 \mathrm{~A}$ includes both transgenic and nontransgenic animals, as it is not possible to distinguish $\operatorname{lin}-15(-)$ and $\operatorname{lin}-15(+)$ animals at the dauer stage. This is the case for both the test and the control strain.

For daf-2(-) dat-1(-) mutant dauers, we checked that the morphology of the pharynx, cuticle, and gonad were those of a fully formed dauer larva, and found that they were. Also, dat-1 mutations did not impair the ability of worms to respond to dauer pheromone, and dat-1(-) dauers formed after exposure to dauer pheromone were also more motile than wild-type dauers. These observations indicate that there is no reason to think dat-1 mutations impair dauer formation, or that their effect is very specific to daf-2/InsR mutant dauers.

Visual assay: adults. Animals were raised until the $\mathrm{L} 4$ stage at $15^{\circ} \mathrm{C}$, and then moved to $25^{\circ} \mathrm{C}$ on plates with E. coli, and scored $3 \mathrm{~d}$ later. CF2625 daf-2(e1370); lin-15(n765ts); akEx248[Pdat-1::gfp; lin-15(+)] and CF2626 daf-2(e1370); lin-15(n765ts); akEx387[Pdat-1::gfp; Pdat-1::hICE; lin-15(+)] were grown at $20^{\circ} \mathrm{C}$, instead of $15^{\circ} \mathrm{C}$, to pick out transgenic animals. Animals were scored as moving if they moved at least one body bend forward or backward. If animals were rollers (see Fig. 5C; supplemental Fig. S3, available at www.jneurosci.org as supplemental material), 
they were scored as moving if their head moved forward or backward in a circle. A minimum of 30 animals per strain was scored in each repeat of the experiment. In all experiments, each strain was tested a minimum of six times. Experiments in Figures $1 C, 5 A, 6 E$, and $7 D$ were part of a set of experiments done in parallel, in which each strain was scored at least 12 times.

For the longitudinal studies (supplemental Fig. $\mathrm{S} 1 A, B$, available at www.jneurosci.org as supplemental material), animals were grown at $15^{\circ} \mathrm{C}$, shifted to $25^{\circ} \mathrm{C}$ as $\mathrm{L} 4$, and scored every day. On the third day, they were shifted back to $15^{\circ} \mathrm{C}$ and scored every day for 2 more days. Each strain was tested four times.

Adult assays using the worm tracker ("Tracker assay"). The parallel worm tracker platform developed by the laboratory of Miriam Goodman (Stanford University, Stanford, CA) was used to characterize adult movement as described previously (Ramot et al., 2008). Animals were grown in the same conditions used for the visual assays, and tested $3 \mathrm{~d}$ after the L4 stage. At the beginning of the experiment, animals were transferred on NGM plates seeded with E. coli OP50 as described previously (Ramot et al., 2008). The only exceptions were the "off food" assays, in which animals were transferred to unseeded plates instead. One minute videos were taken every $10 \mathrm{~min}$ for $90 \mathrm{~min}$ and analyzed using the parallel worm tracker tools (Ramot et al., 2008). The "identify paralyzed" function was used to determine the average speed of the population and the fraction of animals that were moving. The average speed was defined as the average of the instantaneous speed measurement for all tracks in a given video (Ramot et al., 2008). To determine the fraction of animals moving, animals were deemed "paralyzed" if $80 \%$ of the instantaneous speed measurements collected during the track were $<15 \mu \mathrm{m} / \mathrm{s}$. Each strain was tested four to six times. For the tracking assay (see Fig. $6 A$; supplemental Fig. S4, available at www.jneurosci.org as supplemental material), CF3248 daf-2(e1370); muIs176[Pdat-1::gfp; Pdat-1::hICE; Podr-1::rfp] adults were used as daf-2(-) without dopamine neurons. These animals also moved more in a visually scored adult assay (data not shown).

We noticed that, right after the transfer $(t=0 \mathrm{~min})$, more animals moved, and at a faster speed. Over time, both the speed and the percentage of animals moving decreased, and reached a stable value. We considered the initial movement response to be movement in response to a stimulus, whereas we reasoned that the stable level of locomotion represented baseline (unstimulated) movement.

\section{Long-term dopamine addition}

Dopamine plates were prepared as previously described (Schafer and Kenyon, 1995; Chase et al., 2004). A final concentration of $30 \mathrm{~mm}$ dopamine hydrochloride (Sigma-Aldrich) was used. Both $30 \mathrm{~mm}$ and control ("0 $\mathrm{mm}$ ") dopamine plates contained a final concentration of $2 \mathrm{~mm}$ glacial acetic acid. Animals were grown at $15^{\circ} \mathrm{C}$ and shifted to $25^{\circ} \mathrm{C}$ as $\mathrm{L} 4$ larvae. Three days later, animals were moved to dopamine or control plates seeded with E. coli OP50 for $2.5 \mathrm{~h}$. They were then shifted to fresh plates also seeded with $E$. coli containing the same dopamine concentrations and tested using the parallel worm tracker. One minute videos were taken every $10 \mathrm{~min}$ for $60 \mathrm{~min}$. Each strain-concentration combination was tested at least three times.

\section{Dopamine-induced paralysis}

Acute dopamine treatment was performed as described previously (Schafer and Kenyon, 1995), with minor modifications. Animals were grown at $15^{\circ} \mathrm{C}$, shifted to $25^{\circ} \mathrm{C}$ as L4 larvae, and tested the following day. They were transferred to unseeded 1.5\% Difco agar (BD Biosciences) plates containing $2 \mathrm{~mm}$ glacial acetic acid and the indicated concentration of dopamine, and kept at $25^{\circ} \mathrm{C}$ for $1 \mathrm{~h}$. The fraction of animals moving was then determined visually using a dissecting microscope.

\section{Pumping}

Animals were grown at $15^{\circ} \mathrm{C}$ and transferred to $25^{\circ} \mathrm{C}$ at L4 stage. Pumping rates were scored $3 \mathrm{~d}$ later. The contractions of the pharynx in a $30 \mathrm{~s}$ interval were counted for five animals per strain on 3 separate days. Animals were counted whether or not they pumped, as $20 \%$ of the daf2(e1370) mutant animals were observed not to pump at all.

\section{HPLC analysis}

Animals were grown at $15^{\circ} \mathrm{C}$ until the $\mathrm{L} 4$ stage and shifted to $25^{\circ} \mathrm{C}$ for $3 \mathrm{~d}$. Samples were collected in M9 buffer and frozen immediately. HPLC analysis was done by the Neurochemistry Core facility at the Center for Molecular Neuroscience of Vanderbilt University (Nashville, TN). Each strain was tested at least three times.

\section{Statistics}

Statistical analysis was performed using XLSTAT 8.0 add-in software for Excel (Addinsoftware). Appropriate tests were chosen as follows: (1) Student's $t$ test was used when two strains were compared with each other in visual assays; (2) one-way ANOVA followed by post hoc Dunn-Sidak's corrected $t$ test or Dunnet's corrected $t$ test (for comparison with a single control) was used when multiple strains were compared with each other in visual assays (the correction for multiple comparisons was applied for each figure panel separately); (3) two-way ANOVA was used to analyze the tracker data, setting as variables time and strain, time and condition for the on-off food comparisons or time and concentration for the experiments with dopamine addition; $p$ values reported are for the strain/ condition/dopamine concentration component of the analysis (the time component had, as expected, a significant effect in all comparisons); when single data points are reported (e.g., for $t=0 \mathrm{~min}$ ), Student's $t$ test or Dunn-Sidak's corrected $t$ test was used; (4) Kruskal-Wallis test with Dunn's test was used for the pumping data, as these data were not normally distributed.

\section{Results}

\section{Dauer larvae move less than nondauer animals}

C. elegans dauer larvae share certain features with animals in hibernation, as both are states of dormancy characterized by suppressed metabolism and increased resistance to stress (Carey et al., 2003). Consistent with this, Cassada and Russell (1975) reported that dauer larvae have reduced spontaneous locomotion but respond to stimuli. We tested whether we could quantify the quiescence of dauer larvae by observing nondauers and dauers visually and comparing the fractions of animals that moved. We found that this was the case: whereas $56-60 \%$ of nondauer, wildtype animals moved freely, only $14 \%$ of the dauer larvae did (Fig. $1 A$ ). (Henceforth, we refer to this method of scoring as the "visual assay.")

Several signaling pathways interact to regulate dauer formation. In addition to the insulin/IGF- 1 signaling pathway, a TGF- $\beta$ pathway and a cGMP-dependent pathway also influence dauer formation, and mutations in either of these pathways can cause constitutive dauer formation (for review, see Riddle and Albert, 1997; Hu, 2007). To test whether the dauer quiescence phenotype was specific to $d a f-2 /$ InsR mutant dauers, we assayed the movement of dauer larvae formed in response to mutations in daf-7, a gene encoding a TGF- $\beta$ ligand (Ren et al., 1996), and in daf-11, a gene encoding a guanylate cyclase (GC) that acts in the cGMP pathway (Birnby et al., 2000). Dauers caused by all three mutations were quiescent (Fig. $1 B$ ). We also examined wild-type dauers induced by exposure to dauer pheromone, which is a natural cue that induces dauer formation, and confirmed that these animals were quiescent as well (data not shown). We conclude that locomotory quiescence is a general feature of dauer larvae.

\section{daf-2(e1370) adults display a quiescent behavior reminiscent of dauer larvae}

To better characterize the quiescence phenotype, we sought to examine quiescence independently of dauer formation. To this end, we took advantage of the dauer-like locomotory phenotype produced by class II mutations in $d a f-2 /$ InsR, which are thought to cause a strong reduction in insulin/IGF-1-like signaling (Gems 

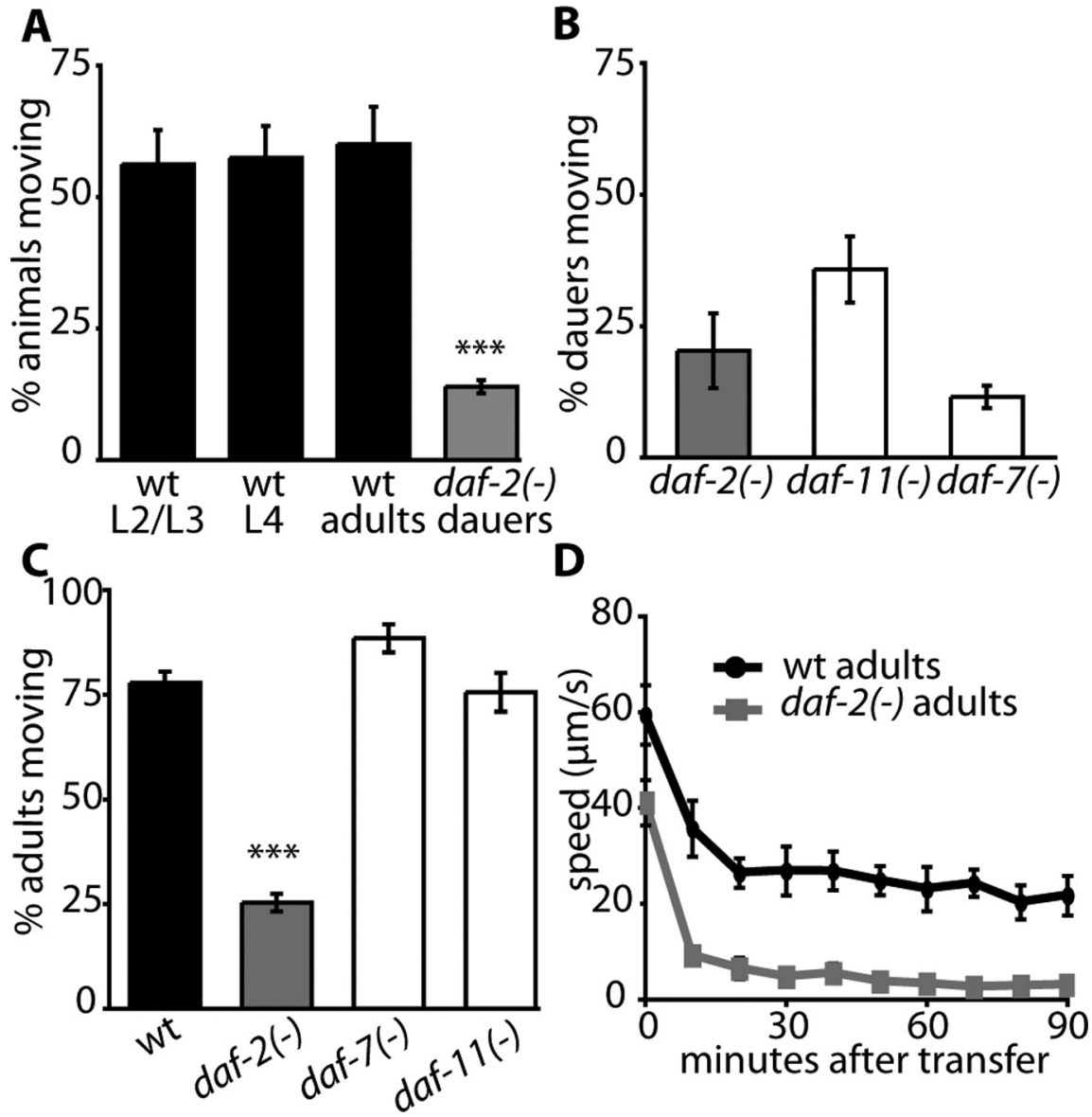

Figure 1. Dauer larvae have reduced spontaneous movement and mutations in the daf-2/InsR pathway can produce dauerlike quiescence in adult animals. $\boldsymbol{A}$, The percentage of daf-2(e1370) dauer larvae that moved on visual observation was compared with that of wild-type (wt) larvae and adults also grown at $25^{\circ} \mathrm{C}$. Percentage moving $\pm \mathrm{SEM}$ : wild-type L2/L3 larvae, $56 \pm 6 \%$; wild-type L4 larvae, $57 \pm 6 \%$; wild-type adults, $60 \pm 7 \%$; daf-2(-) dauer larvae, $14 \pm 1 \%$. ${ }^{* * *} p<0.0001$ for daf-2(-) versus wt (any stage), Dunn-Sidak's corrected $t$ test. $\boldsymbol{B}$, The movement of dauer larvae formed at $25^{\circ} \mathrm{C}$ because of mutations in genes representative of the three major dauer formation pathways was assayed: the insulin/IGF-1 receptor homolog (InsR) daf-2 for the insulin/IGF-1-like pathway, the TGF- $\beta$ homolog daf-7 for the TGF- $\beta$ pathway, and the GC daf- 11 for the cGMP pathway. Dauers formed by all three mutations were quiescent. Percentage moving \pm SEM: daf-2(e1370), $20 \pm 6 \%$; daf-11(sa195), $36 \pm 6 \%$; daf-7(e1372), $12 \pm 2 \%$.C, Mutations in daf-2/InsR, but not mutations in daf-7/TGF- $\beta$ or in daf-11/GC, decreased the percentage of adult animals that moved. Percentage moving \pm SEM: wt, $78 \pm 3 \%$; daf-2(e1370), $25 \pm 2 \%$; daf-7(e1372), $88 \pm 2 \%$; daf-11(sa195), $76 \pm 5 \%$. ${ }^{* * *} p<0.0001$ versus wt, Dunn-Sidak's corrected $t$ test. $\boldsymbol{D}$, daf-2(e1370) adults were compared with wild-type animals using the tracker. Both animals responded to stimulation, but the average baseline speed of the population was lower in daf-2(e1370) adults. $p<0.0001$ (two-way ANOVA) for daf-2(-) versus wt. Error bars represent SEM. Animals in Figure $1, C$ and $D$, were grown at $15^{\circ} \mathrm{C}$, shifted to the quiescence-inducing temperature, $25^{\circ} \mathrm{C}$, at the $L 4$ stage (after the dauer-decision point), and tested at day 3 of adulthood on food.

et al., 1998). Because the process of dauer formation is facilitated by high temperature, these mutations cause constitutive dauer formation at high temperature $\left(25^{\circ} \mathrm{C}\right)$. However, when raised at low temperature $\left(15^{\circ} \mathrm{C}\right)$ until the last stage of larval development and then shifted to $25^{\circ} \mathrm{C}$, these animals develop into adults but have several phenotypes that are reminiscent of dauers, including reduced locomotion. We confirmed that daf-2(e1370) mutant adults progressively became quiescent when shifted to $25^{\circ} \mathrm{C}$ at the last stage of larval development, the L4 stage (Gems et al., 1998) (supplemental Fig. S1 A, available at www.jneurosci.org as supplemental material). (All subsequent adult assays were performed $3 \mathrm{~d}$ after the shift to $25^{\circ} \mathrm{C}$.) The defect could be readily reversed by shifting the animals back to $15^{\circ} \mathrm{C}$ (Gems et al., 1998) (supplemental Fig. S1 B, available at www.jneurosci.org as supplemental material). We also found that daf-2(e1370) animals had dramatically reduced rates of pharyngeal pumping $3 \mathrm{~d}$ after the shift to the restrictive temperature (supplemental Fig. S1C, available at www. jneurosci.org as supplemental material), which is reminiscent of the lack of pumping in dauer larvae. These dauer-like phenotypes can be uncoupled from the increased longevity of daf-2(-) animals, as weaker (class I) mutations in daf-2/InsR do not affect movement but still increase the life span of the worms (Gems et al., 1998).

Like class I daf-2/InsR mutations, mutations in genes from other dauerformation pathways did not cause dauerlike phenotypes as adults. For example, both $d a f-7 /$ TGF- $\beta$ and $d a f-11 /$ GC mutant adults moved as much as wild-type animals (Fig. 1C) and had pumping rates similar to wild-type animals (supplemental Fig. S1C, available at www.jneurosci.org as supplemental material). Although it is possible that changing both the cGMP and the TGF- $\beta$ pathways in the same animal would trigger adult quiescence [these double mutants have an enhanced dauerconstitutive phenotype (Thomas et al., 1993)], the adult data suggest that the daf2/InsR pathway may have a direct effect on the expression of dauer-like quiescence.

Because of their small size, dauer larvae are difficult to track with automated systems. However, we were able to use an automated tracker (Ramot et al., 2008) to analyze the quiescence of daf-2(e1370) adults. (We will refer to assays done using the automated tracker as "tracker assays.") Tracker assays allowed us to observe both the average speed and the percentage of animals moving over an extended period of time.

We noticed that, regardless of the strain, we could now clearly distinguish two stages of movement (Fig. 1D; supplemental Fig. S1 $D$, available at www.jneurosci.org as supplemental material). When first transferred to the assay plate, the animals moved faster, but later the average speed of the animals and the percentage that were moving decreased and subsequently remained more or less constant. We reasoned that the first stage represented movement in response to stimulation (transferring to new plates) (see Materials and Methods), whereas the second stage represented baseline, unstimulated movement. In principle, mutations that alter movement rates could affect the stimulated movement, the baseline movement, or both. We found that daf-2/InsR mutations affected baseline rates of movement, because $60 \mathrm{~min}$ after the beginning of the assay the average speed of the daf-2(-) population had dropped to $3.3 \pm 0.5 \mu \mathrm{m} / \mathrm{s}$, whereas the speed of wild-type animals was $23.1 \pm 4.6 \mu \mathrm{m} / \mathrm{s}$ (Fig. $1 D)(t=60 \mathrm{~min} ; p<0.05$, Dunn-Sidak's corrected $t$ test). In contrast, wild-type and daf-2(-) animals both moved much faster immediately after stimulation (Fig. 1D) [average population speed \pm SEM at $t=0 \mathrm{~min}$ : wt, $59.4 \pm 6.2$ $\mu \mathrm{m} / \mathrm{s}$, vs daf-2(-), $41.1 \pm 4.7 \mu \mathrm{m} / \mathrm{s} ; p<0.05$, Dunn-Sidak's 
corrected $t$ test], although wild-type animals still moved faster than did daf-2/InsR mutant animals. In addition, in both populations almost all the animals were classified as moving (supplemental Fig. S1 $D$, available at www.jneurosci.org as supplemental material) [percentage moving \pm SEM at $t=0 \mathrm{~min}: \mathrm{wt}, 88 \pm 6 \%$, vs daf-2(-), $90 \pm 6 \%$ ]. These data indicate that daf-2/InsR mutant adults, like dauers, are capable of moving after stimulation, consistent with the interpretation that their quiescence is likely attributable to an active modulation of the locomotory circuit, rather than to permanent defects in muscles or neurons or to a debilitating lack of energy.

\section{Mutations that affect dopamine can reverse the quiescence of dauer larvae}

Previous studies have shown that dopamine modulates the locomotion of C. elegans (Schafer and Kenyon, 1995; Sawin et al., 2000; Chase et al., 2004; Hills et al., 2004). Therefore, we asked whether changes in dopamine signaling could affect dauer movement. To test the effect of decreased dopamine signaling, we examined a strain in which dopamine-producing neurons were genetically ablated by cell-specific expression of a cell death caspase (Hills et al., 2004). To test the effects of increased dopamine signaling, we examined animals with a loss-of-function mutation in the dopamine-reuptake transporter dat-1 (Nass et al., 2002), which is needed for termination of the dopamine signal (Gainetdinov and Caron, 2003). In mammals, dopamine transporter (DAT) mutations increase extracellular dopamine levels (Giros et al., 1996), and previous behavioral analysis in C. elegans suggests this is likely to be the case in the worm too (Kindt et al., 2007; McDonald et al., 2007). Neither treatment appeared to affect the ability of the animals to form dauers (data not shown) (see Materials and Methods). Surprisingly, we found that both conditions stimulated dauer movement: daf-2(-) dauers with no dopamine-producing neurons moved more than control daf2(-) dauers (Fig. 2A) and daf-2(-) dat-1(-) double-mutant dauers moved more than daf-2(-) dauers (Fig. $2 B$ ). We also found that dat-1 mutations could increase movement in dauers obtained by exposure to dauer pheromone (data not shown). Together, these data suggest that dopamine may influence two pathways that stimulate dauer movement, one activated by reduced dopamine signaling and the other activated by increased dopamine signaling.

Decreases in dopamine levels in C. elegans have previously been correlated with increased locomotion in the absence of food (Sawin et al., 2000), which could potentially explain why loss of dopamine-producing neurons can increase movement in dauer larvae. In contrast, increases in dopamine signaling have not been reported to stimulate locomotion in C. elegans before. Therefore, we will discuss the effects of mutations predicted to increase or decrease dopamine-signaling levels separately.

\section{dat-1 mutations stimulate the movement of daf-2(e1370) adults}

To study the effects of increasing dopamine signaling, we first asked whether dat-1 mutations could also affect the quiescence of daf-2/InsR mutant adults. We found that dat-1 mutations dramatically increased the percentage of $d a f-2(-)$ animals moving in the visual assay [percentage moving \pm SEM: daf-2(e1370), $25 \pm 2 \%$; daf-2(e1370) dat-1(ok157), $67 \pm 3 \%$; $p<0.0001$, Student's $t$ test].

When we characterized these mutants further using the tracker assay, we found that dat-1 mutations caused an increase in movement after the animals were transferred to the assay

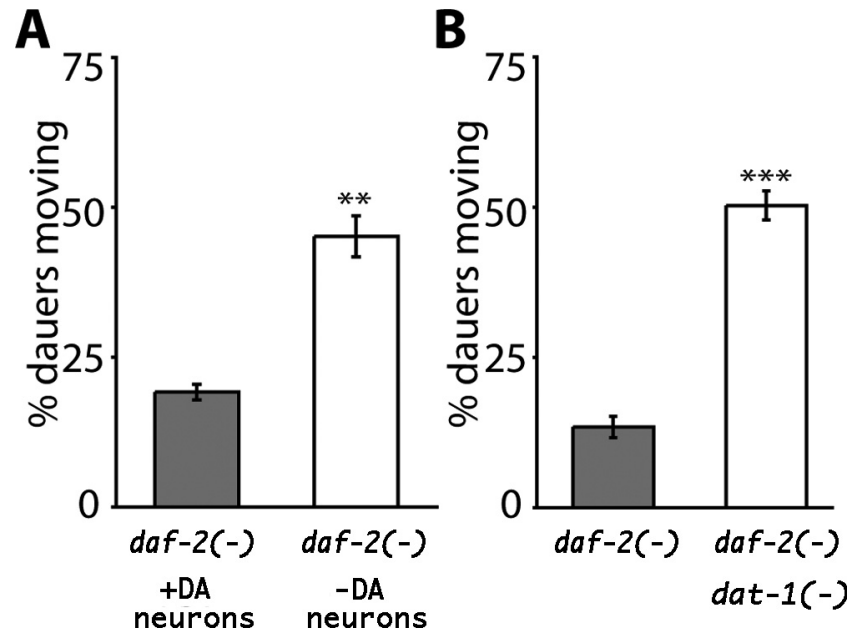

Figure 2. Ablation of the dopamine-producing neurons and mutations in the dopamine transporter dat- 1 increase the percentage of moving dauers. $\boldsymbol{A}$, daf-2(e1370) strains expressing GFP (+DA neurons) or a human homolog of $C$. elegans caspase (interleukin-converting enzyme) (-DA neurons) in the dopamine-producing neurons (Hills et al., 2004) were tested to examine the effect of genetically ablating dopamine-producing neurons. Loss of the dopamineproducing neurons increased the number of dauers that moved. Percentage moving $\pm \mathrm{SEM}$ : daf-2(-); lin-15(-); akEx248[Pdat-1::gfp; lin-15(+)] [daf-2(-) + DA neurons], $19 \pm 3 \%$; daf-2(-); lin-15(-); akEx387[Pdat-1::gfp; Pdat-1::hICE; lin-15(+)][daf-2(-) -DA neurons], $45 \pm 5 \%$. ${ }^{* *} p<0.01$, Student's $t$ test. $\boldsymbol{B}$, The effect of loss of the dopamine transporter was tested by comparing spontaneous movement in daf-2(e1370) and daf-2(e1370) dat1(ok157) dauer larvae. dat-1 mutations increased the percentage of dauers that were moving. Percentage moving \pm SEM: daf-2(-), $14 \pm 2 \%$; daf-2(-) dat-1(-), $50 \pm 3 \%$. ${ }^{* *} p<$ 0.0001 , Student's $t$ test. Error bars represent SEM.

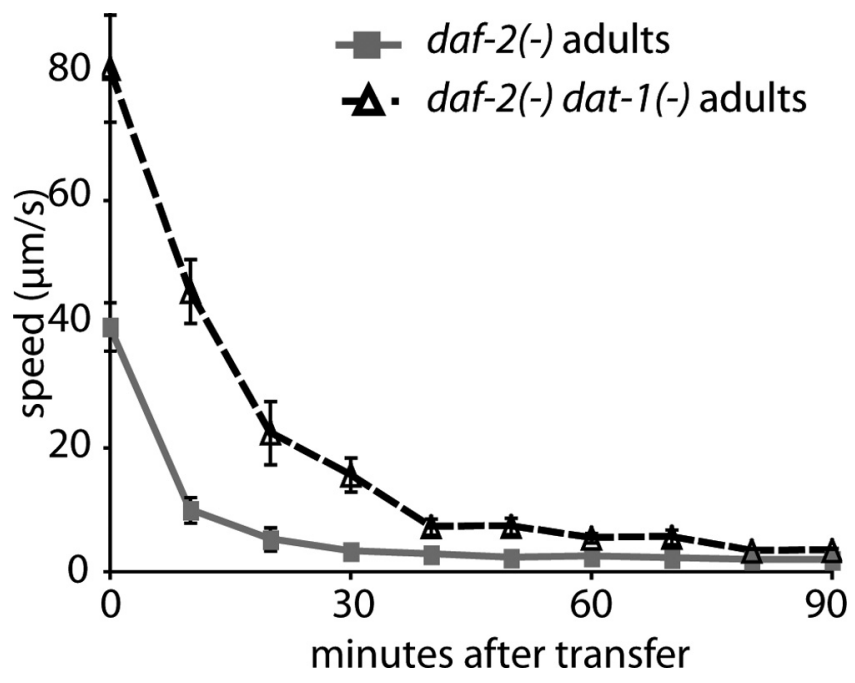

Figure 3. Mutations in dat- 1 increase movement in daf-2/InsR mutant adults by affecting their locomotory response to stimulation. When stimulated, daf-2(e1370) dat-1(ok157) animals initially moved faster than daf-2(e1370) mutants alone. $p<0.0001$, two-way ANOVA. Error bars represent SEM. All animals were grown at $15^{\circ} \mathrm{C}$, shifted to $25^{\circ} \mathrm{C}$ at the $\mathrm{L} 4$ stage, and tested at day 3 of adulthood on food.

plates, suggesting that increasing dopamine signaling affected movement in response to stimulation. However, by $40 \mathrm{~min}$, most of the animals had stopped moving, suggesting that dat-1 mutations did not affect baseline movement of daf-2(-) animals (Fig. $3)$. Specifically, at time 0 , dat- 1 mutations dramatically increased the average speed of the $d a f-2(-)$ population [average speed \pm SEM at $t=0 \mathrm{~min}$ : daf $-2(-), 39.5 \pm 3.8 \mu \mathrm{m} / \mathrm{s}$ vs daf- $2(-)$ dat$1(-), 81.3 \pm 8.6 \mu \mathrm{m} / \mathrm{s} ; p<0.01$, Dunn-Sidak's corrected $t$ test], 

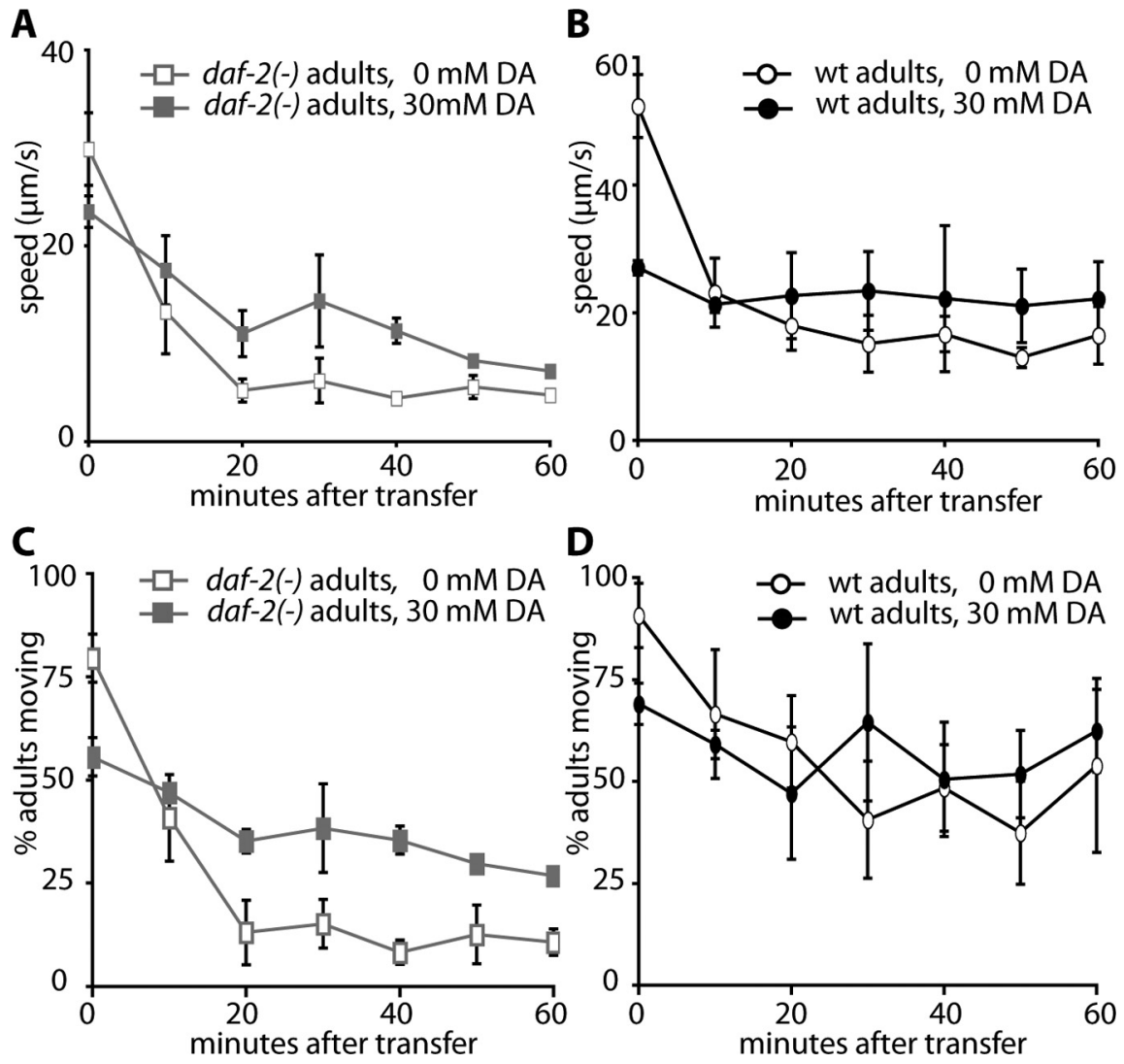

Figure 4. Long-term exogenous addition of dopamine suppresses quiescence in daf-2/InsR mutant animals. daf-2(e1370) (A, $\boldsymbol{C}$ ) and wild-type (wt) $(\boldsymbol{B}, \boldsymbol{D})$ animals were incubated on plates containing 0 or $30 \mathrm{~mm}$ dopamine for $3.5 \mathrm{~h}$ ( $2.5 \mathrm{~h}$ of preincubation and $1 \mathrm{~h}$ of recording). Exogenous dopamine increased the average speed $(\boldsymbol{A})(p<0.05$, two-way ANOVA) and the percentage of animals moving $(\boldsymbol{C})(p<0.001$, two-way ANOVA) in daf-2/InsR mutant adults but had no effect on the speed $(\boldsymbol{B})(p=0.8$, two-way ANOVA) and percentage of animals moving $(\boldsymbol{D})(p=0.9$, two-way ANOVA) in wild-type adults. All animals were grown at $15^{\circ} \mathrm{C}$, shifted to $25^{\circ} \mathrm{C}$ at the $\mathrm{L} 4$ stage, and tested at day 3 of adulthood on food. Error bars represent SEM.

but the difference decreased over time [average speed \pm SEM at $t=90 \mathrm{~min}:$ daf-2(-), $2.8 \pm 0.1 \mu \mathrm{m} / \mathrm{s}$, vs daf-2(-) dat-1(-), $3.4 \pm 0.3 \mu \mathrm{m} / \mathrm{s} ; p<0.01$, Dunn-Sidak's corrected $t$ test]. We conclude that dat-1 mutations, presumably by increasing dopamine signaling, allow daf-2/InsR mutant adults to respond more vigorously to external stimuli.

\section{dat-1 mutations have little effect on wild-type movement}

Finding that mutations predicted to increase dopamine signaling increased movement in response to stimulation was surprising, because in $C$. elegans loss of dopamine is known to increase movement in the presence of food (Sawin et al., 2000). However in other organisms, mutations in genes encoding DAT, which prevent reuptake of the neurotransmitter and thus prolong dopamine signaling, cause hyperactivity (Giros et al., 1996; Kume et al., 2005). dat-1 mutations in C. elegans have been reported not to have an effect on wild-type locomotion under standard culture conditions (McDonald et al., 2007). To investigate this further, we tested what effect dat-1 mutations had on wild-type movement rates in the tracker assay. We found that dat-1 mutations did not change the average population speed of $d a f-2(+)$ worms (supplemental Fig. S2A, available at www.jneurosci.org as supplemental material), despite their dramatic effect on the speed of daf-2(-) animals (Fig. 3). dat-1 mutations did increase the percentage of daf-2(+) animals moving after stimulation (supplemental Fig. S2C, available at www.jneurosci.org as supplemental material) (a measure that is clearly visible only with the tracker); however, the effect was relatively modest [supplemental Fig. S2C (available at www. jneurosci.org as supplemental material), percentage moving \pm SEM at time $t=20$ min: wt, $62 \pm 4 \%$, vs dat $-1(-), 77 \pm 5 \%$; compare with supplemental Fig. S5A (available at www.jneurosci.org as supplemental material), percentage moving \pm SEM at time $t=20 \mathrm{~min}$ : daf $-2(-), 10 \pm 6 \%$, vs daf2(-) dat-1(-), $46 \pm 10 \%]$.

Exogenous dopamine stimulates the movement of daf-2/InsR mutant adults These data suggest that a prolonged increase in dopamine signaling can stimulate the movement of $C$. elegans, particularly that of quiescent insulin/IGF-1-receptor mutants. We decided to test this more directly by asking whether we could mimic the effect of the dat-1 mutation by prolonged exposure to exogenous dopamine. We found that exposing the worms to 30 $\mathrm{mm}$ dopamine (DA) for $3.5 \mathrm{~h}$ (which included a $2.5 \mathrm{~h}$ preexposure and $1 \mathrm{~h}$ of recording on the tracker) increased the movement of quiescent daf-2(-) adults, both in terms of the average speed of the population (Fig. 4A) and the percentage of worms moving (Fig. 4C). No significant effect was detected in wild-type animals (Fig. $4 B, D$ ). We conclude that prolonged exposure to dopamine increases the movement of quiescent daf-2/InsR mutant adults.

Notably, right after stimulation, at time 0 , wild-type animals treated with dopamine actually moved less than control-treated animals (Fig. 4D) (percentage moving $\pm \mathrm{SEM}$ at $t=0 \mathrm{~min}: 0 \mathrm{mM} \mathrm{DA}, 91 \pm 11 \%$, vs $30 \mathrm{~mm}$ DA, $69 \pm 7 \%$; $p<0.05$, Student's $t$ test), a decrease that may reflect the paralysis reported previously in acute assays (Schafer and Kenyon, 1995; Chase et al., 2004).

In wild-type animals treated with $30 \mathrm{~mm}$ dopamine, we observed a trend toward increased speed, but it was not statistically significant. We considered the possibility that we failed to see an effect on wild-type animals because their average speed was already high. However, food removal could double the speed of wild-type animals (supplemental Fig. S2 A, B, available at www. jneurosci.org as supplemental material). Therefore, these data indicate that increases in dopamine specifically suppress the quiescence of daf-2/InsR mutant animals after stimulation.

\section{daf-2/InsR mutations require daf-16/FOXO activity in neurons to affect movement}

daf-2/InsR mutations lead to increased nuclear localization and activation of the FOXO transcription factor DAF-16 (Henderson and Johnson, 2001; Lee et al., 2001; Lin et al., 2001). daf-16/ FOXO is required for daf-2/InsR mutant animals to become dauers (Riddle et al., 1981; Vowels and Thomas, 1992; Gottlieb and Ruvkun, 1994), and Gems et al. (1998) showed that daf-16/ FOXO is also required for the quiescence phenotype of daf2(e1370) adults. Therefore, we wanted to test whether daf-16/ FOXO was required for the enhanced response of $d a f-2 /$ InsR mutants to dat-1 mutations. 
First, we confirmed that daf-16/FOXO mutations increased movement in daf2(e1370) adults (Fig. 5A). When we analyzed daf-16(-); daf-2(-) double mutants with the tracker, we found that daf$16 /$ FOXO mutations partially rescued the decreased baseline movement of daf-2/ InsR mutant adults (Fig. 5B). At $t=60$ min, when the worms have reached a stable speed, the average speed of wild-type animals was $23.1 \pm 4.6 \mu \mathrm{m} / \mathrm{s}$, whereas that of daf-2(-) animals was $3.3 \pm 0.5 \mu \mathrm{m} / \mathrm{s}$. In contrast, daf-16(-); daf-2(-) mutants moved at $12.5 \pm 2.2 \mu \mathrm{m} / \mathrm{s}$. We conclude that, although there may be additional pathways involved, DAF-16/FOXO has a role in controlling quiescence in response to changes in insulin/IGF-1 signaling.

We then asked in which tissue DAF-16/ FOXO acts to prevent animals from moving. To this end, we used transgenic lines expressing a daf-16 cDNA under the control of tissue-specific promoters in a daf16(-); daf-2(-) mutant background. Libina et al. (2003) previously showed that neuronal daf-16/FOXO expression is sufficient to restore a constitutive dauerformation phenotype to daf-16(-); daf2(-) double mutants. As expected, expression of daf-16/FOXO under its own promoter restored the quiescence phenotype of the daf-2/InsR mutant adults. Expression from the neuronal promoter unc119 also restored the quiescence, whereas expression from intestinal (Pges-1) and muscle (Pmyo-3) promoters did not (Fig. $5 C$ ). These data suggest that DAF-16/ FOXO acts in neurons to slow the movement of daf-2/InsR mutants. We noticed that expression from the pan-neuronal promoter did not restore quiescence as well as expression from the daf-16 promoter did. It is possible that DAF-16/ FOXO activity in other tissues contributes to the regulation of quiescence or that the unc-119 promoter we used does not drive sufficient levels of expression to give a complete rescue.

We attempted to further define the neuronal population in which DAF-16/ FOXO acts to regulate dauer-like quiescence by expressing daf-16/FOXO under promoters specific for neuronal subpopulations in daf-16(-); daf-2(-) animals. We created transgenic lines in which daf16/FOXO was expressed from promoters specific for acetylcholinergic (Pacr-2) and GABAergic (Punc-47) motor neurons, a wide range of interneurons (Pglr-5), command interneurons (Pnmr-1), a range of sensory neurons (Posm-5) and dopamineproducing neurons (Pdat-1). We confirmed that GFP::DAF-16 protein was de-
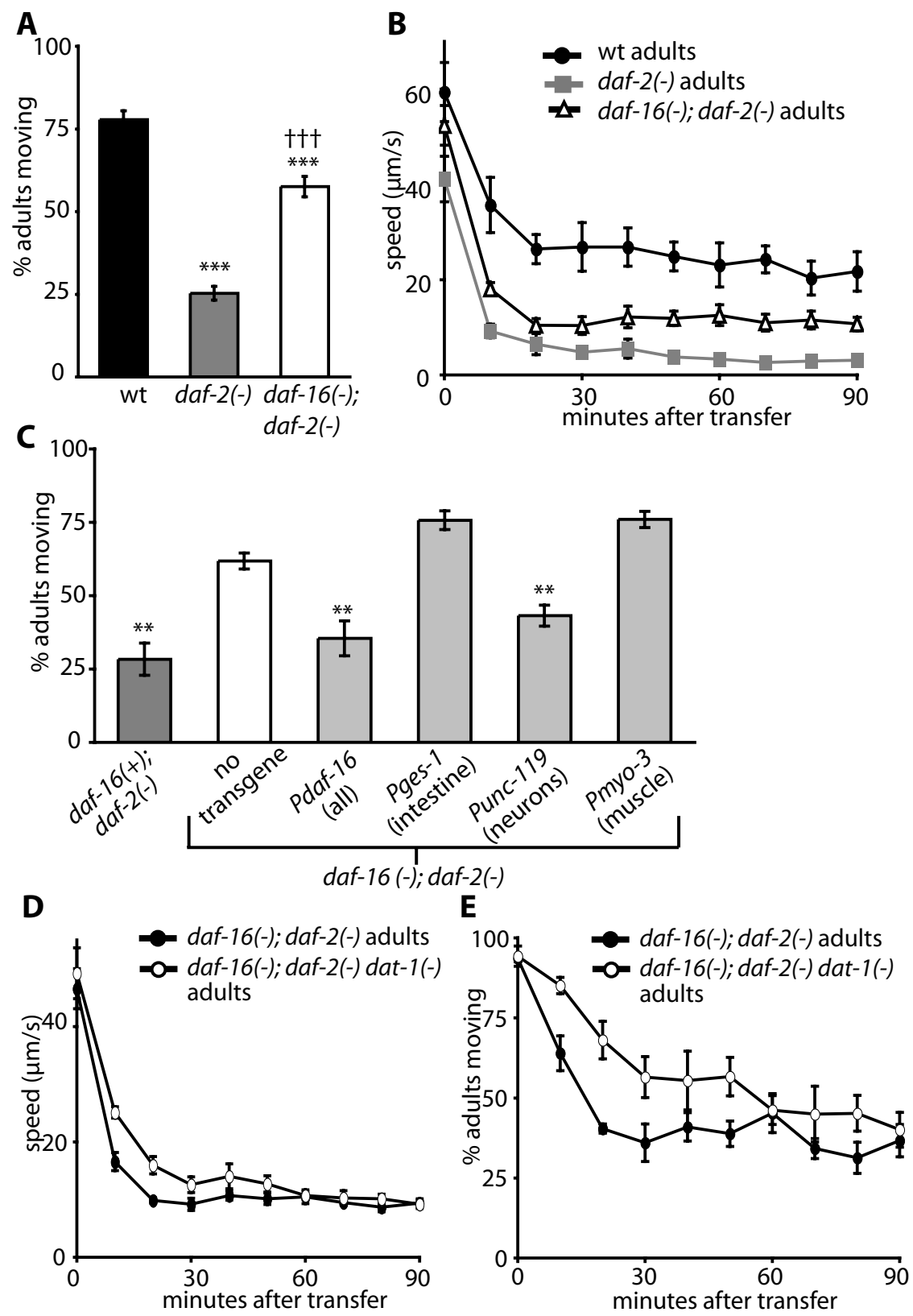

Figure 5. The role of daf-16/F0X0 in the regulation of daf-2/InsR mutant dauer-like quiescence. $\boldsymbol{A}, \boldsymbol{B}$, daf-16(mu86); daf2 (e1370) double-mutant animals were more active than daf-2(e1370) mutants, both in the visual ( $\boldsymbol{A})$ and the tracker $(\boldsymbol{B})$ assays, but were still less active than wt animals. $A$, Percentage moving \pm SEM: wild-type, $77 \pm 3 \%$; daf-2( -$), 25 \pm 2 \%$; daf- $16(-)$; daf- $2(-), 57 \pm 3 \%{ }^{* * *} p<0.0001$ versus wt control, ${ }^{\dagger+t} p<0.0001$ versus daf- $2(-)$, Dunn-Sidak's corrected $t$ test. $\boldsymbol{B}$, The average speed of daf-16(mu86); daf-2(e1370) animals was higher than that of daf-2(e1370) animals but lower than that of wild-type animals, suggesting that daf-16/F0X0 mutations can only partially suppress daf-2(e1370) quiescence. $p<0.0001$ (two-way ANOVA) for daf-16(-); daf-2(-) versus daf-2(-) and daf-16(-); daf-2(-) versus wt. Error bars represent SEM. C, Neuronal expression of daf-16/FOX0 was sufficient for daf-2/InsR mutant worms to become inactive. daf-16(-); daf-2(-) animals carrying transgenes expressing daf- 16 cDNA under different promoters were tested against daf-2(-) and daf-16(-); daf-2(-) strains expressing only the coinjection marker rol-6. Expression of daf-16/FOXO under the control of its own promoter or the neuronal unc-119 promoter led to quiescence, whereas intestinal (Pges-1) or muscle (Pmyo-3) expression had no effect. Percentage moving \pm SEM: daf-16(+); daf-2(-), $28 \pm 5 \%$; daf-16(-); daf-2(-), $62 \pm 3 \%$; daf-16(-); daf-2(-); Pdaf-16::gfp:::daf-16, $35 \pm 6 \%$; daf-16(-); daf-2(-); Pges-1::gfp::.daf-16, $76 \pm 3 \%$; daf-16(-); daf-2(-); Punc-119::gfp:::daf-16, $43 \pm 4 \%$; daf-16(-); daf-2(-); Pmyo-3::gfp::daf-16, $76 \pm 3 \% .{ }^{* *} p<0.01$ (Dunnet's corrected $t$ test) versus daf-16(-); daf-2(-). D, E, dat-1(ok157) mutations had a small effect on the speed (D) of daf-16(mu86); daf-2(e1370) animals but increased the percentage of animals moving $(\boldsymbol{E})$. This is similar to the effect that dat-1(ok157) mutations had on wild-type animals. Compare Figure 5D with Figure 3 and supplemental Figure S2 A (available at www.jneurosci.org as supplemental material), and Figure $5 E$ with supplemental Figures $\$ 5 A$ and $S 2 C$ (available at www.jneurosci.org as supplemental material). $D, p<0.001$, two-way ANOVA. $E, p<0.0001$, two-way ANOVA. All animals were grown at $15^{\circ} \mathrm{C}$, shifted to $25^{\circ} \mathrm{C}$ at the $L 4$ stage, and tested at day 3 of adulthood on food. Error bars represent SEM. 

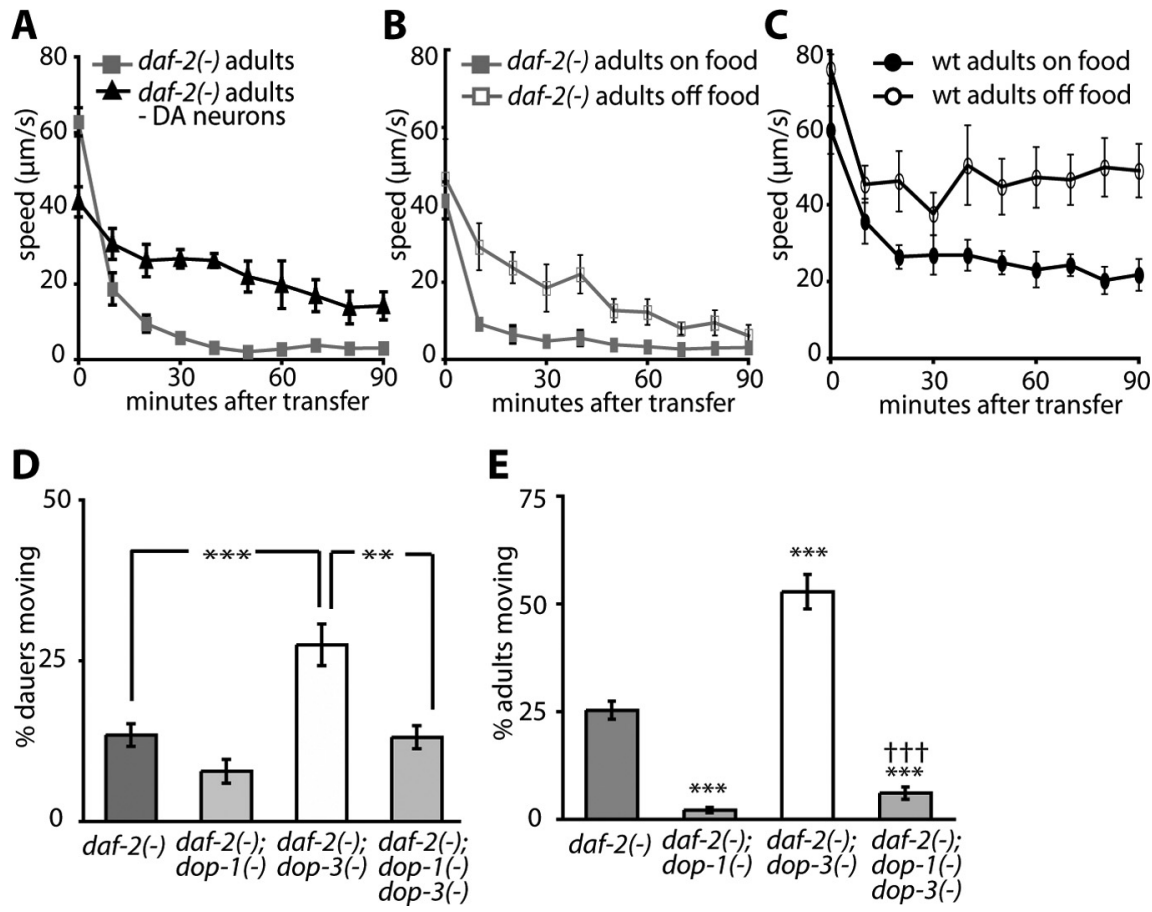

Figure 6. daf-2(e1370) animals display a basal slowing response and are stimulated by mutations that mimic lack of food. $\boldsymbol{A}$, Ablation of the dopamine-producing neurons by expression of a human interleukin-converting enzyme (Hills et al., 2004) in daf-2(e1370) animals [daf-2(-) -DA neurons] increased their movement as adults. $p<0.0001$, two-way ANOVA. $\boldsymbol{B}, \boldsymbol{C}$, daf-2(e1370) (B) and wt (C) animals were transferred to plates with food ("on food") or without food ("off food") and observed for $90 \mathrm{~min}$. The average speed of the worms is plotted as a function of time after transfer. As previously reported for younger wild-type animals (Sawin et al., 2000), the speed of wild-type animals was higher in the absence of food. In daf-2(-) animals, the average speed off food was also higher right after stimulation, but decreased over time. $p<0.0001$ for wild type on food versus off food and daf-2(-) on food versus off food (two-way ANOVA). D, daf-2(e1370); dop-3(vs 106) dauers moved more than daf-2(e1370) dauers, but their increased movement was suppressed by dop-1(vs100) mutations. Percentage moving \pm SEM: daf-2(-), $14 \pm$ 2\%; daf-2(-);dop-1(-), $8 \pm 2 \%$; daf-2(-);dop-3(-), $27 \pm 3 \%$;daf-2(-);dop-1(-) dop-3(-), $13 \pm 2 \%$. *** $<0.0001$ and ${ }^{* *} p<0.01$, Dunn-Sidak's corrected $t$ test. $\boldsymbol{E}$, Mutations in the dopamine receptor dop-3 caused daf-2(e1370) adults to move more. Conversely, mutations in the dopamine receptor dop-1 caused them to move even less. daf-2(e1370); dop-1(vs100) dop3(vs 106) animals moved similarly to daf-2(e1370); dop-1(vs100) animals, suggesting that dop-1 acts downstream of dop-3 in the regulation of daf-2(e1370) adult quiescence. Percentage moving \pm SEM: daf-2(-), $25 \pm 2 \%$; daf-2(-); dop-1(-), $2 \pm 1 \%$; daf-2(-);dop-3(-), $53 \pm 4 \%$; daf-2(-); dop-1(-) dop-3(-), $6 \pm 1 \%{ }^{* * *} p<0.0001$ versus daf-2(-), ${ }^{\text {ttt }} p<0.0001$ for daf-2(-);dop-3(-) versus daf-2(-);dop 1(-) dop-3(-), Dunn-Sidak's corrected $t$ test. All adult animals were grown at $15^{\circ} \mathrm{C}$, shifted to $25^{\circ} \mathrm{C}$ at the $L 4$ stage, and tested at day 3 of adulthood. Error bars represent SEM.

tectable in the expected cells in all the lines we tested. Unlike the pan-neuronal daf-16/FOXO-expressing line, none of these neuron-specific lines showed a significant reduction of movement compared with daf-16(-); daf-2(-) animals (supplemental Fig. S3, available at www.jneurosci.org as supplemental material), although several of them showed a small decrease in the percentage of worms moving. (For more details, see the legends of the supplemental figures, available at www.jneurosci.org as supplemental material.) These data suggest that DAF-16/FOXO may act in more than one type of neuron to regulate dauer-like quiescence; alternatively, it is possible that we have not tested the right neuronal population.

\section{Loss of daf-16/FOXO causes daf-2/InsR mutants to respond more like wild type to dat-1 mutations}

Because daf-16/FOXO and dat-1 mutations both partially suppress the quiescence phenotype of daf-2/InsR mutant adults, and both of them act in neurons, we tested how daf-16(-); daf-2(-) animals behaved when the dat-1 dopamine transporter was removed. We found that the average speed of daf-16(-); daf-2(-) dat-1(-) mutants was only slightly higher than that of daf-
16(-); daf-2(-) mutants (Fig. 5D), which is similar to the situation in wild-type animals (supplemental Fig. S2 A, available at www.jneurosci.org as supplemental material). In addition, as in wild type (supplemental Fig. 2C, available at www. jneurosci.org as supplemental material), a greater percentage of daf-16(-); daf2(-) dat-1(-) triple mutants moved at early time points compared with daf16(-); daf-2(-) double mutants (Fig. $5 E)$. These data indicate that dat-1 mutations affect daf-16(-); daf-2(-) mutants similarly to the way they affect wild type. These data suggest that daf-16/FOXO is necessary for daf-2/InsR mutant animals to respond to increased dopamine signaling with increased movement.

\section{daf-2/InsR mutants have a normal basal slowing response}

Our data indicate that dat-1 mutations and exogenous dopamine addition can stimulate movement in daf-2/InsR mutant dauers and adults. However, as mentioned previously, previous studies of the dopamine system in C. elegans have focused on the animals' requirement for dopamine to slow their movement in response to food, an effect that has been called the "basal slowing response" (Sawin et al., 2000). In this context, reduction of dopamine increases the speed of the animals. We wondered what role this response played in dauer and dauer-like animals. Indeed, we found that reduction in dopamine signaling because of ablation of dopamineproducing neurons also increased the movement of daf-2/InsR mutant dauers (Fig. 2A) and adults [Fig. 6A, as well as by visual scoring: percentage moving \pm SEM: daf-2(-) +DA neurons, $39 \pm 10 \%$; daf$2(-)-$ DA neurons, $80 \pm 3 \%$; $p<0.05$, Student's $t$ test $]$.

We confirmed that the behavioral change seen in daf-2(-) animals with no dopamine neurons is attributable to the loss of dopamine, because when we compared the movement of daf$2(-)$ animals to daf-2(-) animals with no dopamine neurons in the presence of exogenous dopamine $(30 \mathrm{~mm})$, there was no longer any difference between the two strains (supplemental Fig. S4, available at www.jneurosci.org as supplemental material).

To explain the apparent paradox that both increasing and decreasing dopamine signaling leads to increased movement in dauers and dauer-like adults, we hypothesized that there are two different mechanisms at play. On the one hand, for reasons that are not understood, increases in dopamine preferentially stimulate the movement of daf-2/InsR mutant dauers and adults. However, loss of dopamine-producing neurons speeds up daf-2/InsR mutant adults, just as it does wild-type animals, because loss of dopamine mimics the absence of food, which in turn stimulates movement.

To test this hypothesis, we asked whether daf-2/InsR mutant animals have a basal slowing response, that is, whether they move more in the absence of food. We found that this was the case, as 
the average speed of $d a f-2(-)$ adults was higher when the animals were off food than when they were on food (Fig. $6 B$ ). However, daf-2(-) animals still eventually stopped moving in the absence of food (Fig. $6 B$ ) (average population speed \pm SEM at $t=90 \mathrm{~min}$ : on food, $3.1 \pm 0.3 \mu \mathrm{m} / \mathrm{s}$, vs off food, $6.1 \pm 2.7 \mu \mathrm{m} / \mathrm{s} ; p=0.35$, Student's $t$ test), which indicated that absence of food did not alter baseline movement but rather the response of animals to stimuli. Interestingly, loss of the dopamine neurons seemed to have some effect on both the stimulation-induced movement and the baseline movement of the animals (Fig. 6A) [average population speed $\pm \mathrm{SEM}$ at $t=90 \mathrm{~min}$ : daf-2(-), $3.0 \pm 0.1$ $\mu \mathrm{m} / \mathrm{s}$, vs daf-2(-) -DA neurons, $14.2 \pm 3.7 \mu \mathrm{m} / \mathrm{s} ; p=0.05$, Student's $t$ test]. It is thought that food removal reduces dopamine secretion in the animals and that in turn this causes the increase in movement (Sawin et al., 2000). Perhaps baseline movement is not affected in daf-2(-) animals in the absence of food because there is residual dopamine secretion or because dopamine secretion can resume at a later time. In contrast, no dopamine secretion is ever present in animals without dopamine neurons.

Next, we tested whether other mutations in dopaminesignaling genes known to affect the basal slowing response in wild-type animals also affected the locomotion of daf-2/InsR mutant animals. We found that mutations in the dopamine receptor dop-3 (Chase et al., 2004), which prevent slowing in response to food, increased the percentage of daf-2(e1370) dauers and dauerlike adults that were moving (Fig. 6D,E). Furthermore, we found that in both types of animals the increased motility produced by dop-3 mutations was dependent on another dopamine receptor, dop-1 (Fig. $6 D, E$ ). In the basal slowing response of nondauer animals, dop-3 mutations have a similar epistatic relationship with mutations in dop-1 (Chase et al., 2004). In addition, we tested whether DOP-1 and DOP-3 acted in acetylcholineproducing motor neurons to modulate daf-2/InsR mutant quiescence and found that they did: daf-2(-); dop-1(-) adults expressing dop-1 in the motor neurons and daf-2(-); dop-3(-) adults expressing dop-3 in the motor neurons moved as much as daf-2(-) mutants alone (supplemental Fig. S5D, available at www.jneurosci.org as supplemental material). DOP-1 and DOP-3 also act in the motor neurons in the context of the basal slowing response (Chase et al., 2004). Together, these data suggest that dopamine-pathway mutations that prevent the slowing response to food may affect the quiescence of $d a f-2 /$ InsR mutant animals because they mimic food withdrawal. They also suggest that daf-2/InsR-mutant quiescence is not simply an exaggerated response to food, as quiescence is displayed in the absence of food as well. Instead, it appears that a reduction in dopamine signaling can stimulate movement equally in daf-2/InsR mutant animals and in wild-type animals. In contrast, daf-2/InsR mutant animals may respond in a different manner from wild type to increases in dopamine signaling.

\section{DOP-3 receptors may also mediate the effect of increased dopamine on daf-2/InsR mutant quiescence}

Because we showed that loss of some dopamine receptors affects the motility of daf-2/InsR mutant dauer and dauer-like adults, we wanted to examine the relative contributions of the receptors to the reduced locomotion of daf-2(-) animals and to the stimulated locomotion of daf-2(-) dat-1(-) animals. First, we tested animals carrying mutations in all four known C. elegans dopamine receptors [henceforth we will refer to all the dop-2(-); dop-4(-) dop-1(-) dop-3(-) animals as "dop-(1-4)(-)" for simplicity] (Suo et al., 2002, 2003; Chase et al., 2004; Sugiura et al., 2005). Loss of the four receptors, like loss of dopamine neurons, stimulated movement in daf-2(-) animals [compare daf$2(-)$; dop- $(1-4)(-)$ animals with daf-2(-) single mutants (Fig. $7 A)]$. These animals could not be further stimulated to move by increases in dopamine [compare daf-2(-) dat-1(-); dop-(14) (-) animals with daf-2(-); dop-(1-4) animals (Fig. 7A)]. This suggests that one or more of the dopamine receptors identified so far mediates the effect of dat-1 mutation on daf-2/InsR mutant adult quiescence.

In daf-2(-) dauers, loss of the dopamine receptors also decreased the effect that the dat-1 mutation had on movement (Fig. $7 B$ ), although the effect was less dramatic than in dauer-like adults (Fig. 7A). Another important difference between dauers and dauer-like adults was that daf-2(-); dop-(1-4)(-) dauers did not move significantly more than did daf-2(-) single mutant dauers (Fig. $7 B$ ). Because the dop- $(1-4)(-)$ genotype did not produce the same effect as loss of dopamine neurons (compare, for example, Figs. $2 A, 7 B$ ), there may be additional, yet unidentified, dopamine receptors. This interpretation is consistent with another observation we made. Namely, we found that dop- $(1-4)(-)$ animals still became paralyzed in a dopamine-induced paralysis assay (supplemental Fig. S6, available at www.jneurosci.org as supplemental material). Alternative explanations for these data are that some of these mutations may not be null or that one of the receptors has ligand-independent activity.

We then compared daf-2(-) and daf-2(-) dat-1(-) mutant animals that lacked single receptors. Our finding differed slightly between dauer larvae and dauer-like adults (Fig. 7C,D). dop-3 receptor mutations had dramatic effects on both dauers and adult animals, as daf-2(-) dat-1(-); dop-3(-) dauers and adults did not move any more than did daf-2(-); dop-3(-) dauers and adults (Fig. $7 C, D$ ). This suggests that DOP-3 has an important role not only in daf-2/InsR mutant quiescence, as mentioned in the previous section, but also in the stimulation of locomotion caused by increase in dopamine. Conversely, we found that loss of the DOP-2 receptor appeared to no effect on dauer quiescence and a minor effect on adult quiescence; also, it did not prevent the stimulation of movement caused by dopamine (Fig. 7C,D).

The two other receptors, DOP-1 and DOP-4, had striking effects only in the adult animals. dop-4 mutations completely blocked the increased movement of daf-2(-) dat-1(-) adults (Fig. 7D), suggesting that DOP-4 plays an important role. dop-1 receptors further decreased the motility of daf-2(-) adults and daf-2(-) dat-1(-) adults (Fig. 7D). Because the effect of dop-1 mutations on both daf-2(-) animals and daf-2(-) dat-1(-) animals appeared similar in magnitude, we suggest that this effect is independent of any increase in dopamine levels. We do not know the reason for the discrepancy between the effects of dopamine receptor mutations on dauer and adult animals, but these data suggest that there are some significant differences between dauers and dauer-like adults.

\section{Discussion}

In this study, we investigated the effects of dopamine signaling on a behavioral condition reminiscent of hibernation in C. elegans; namely, the quiescence of daf-2/InsR mutant dauer larvae and dauer-like adults. Both types of animals display a remarkable and mostly uncharacterized decrease in spontaneous movement. We found that perturbations in dopamine signaling have dramatic effects on daf-2/InsR mutant dauer and adult movement. Paradoxically, both lack of dopamine-producing neurons, which should prevent dopamine production, and reduced clearance of dopamine from the extracellular medium, which should increase 

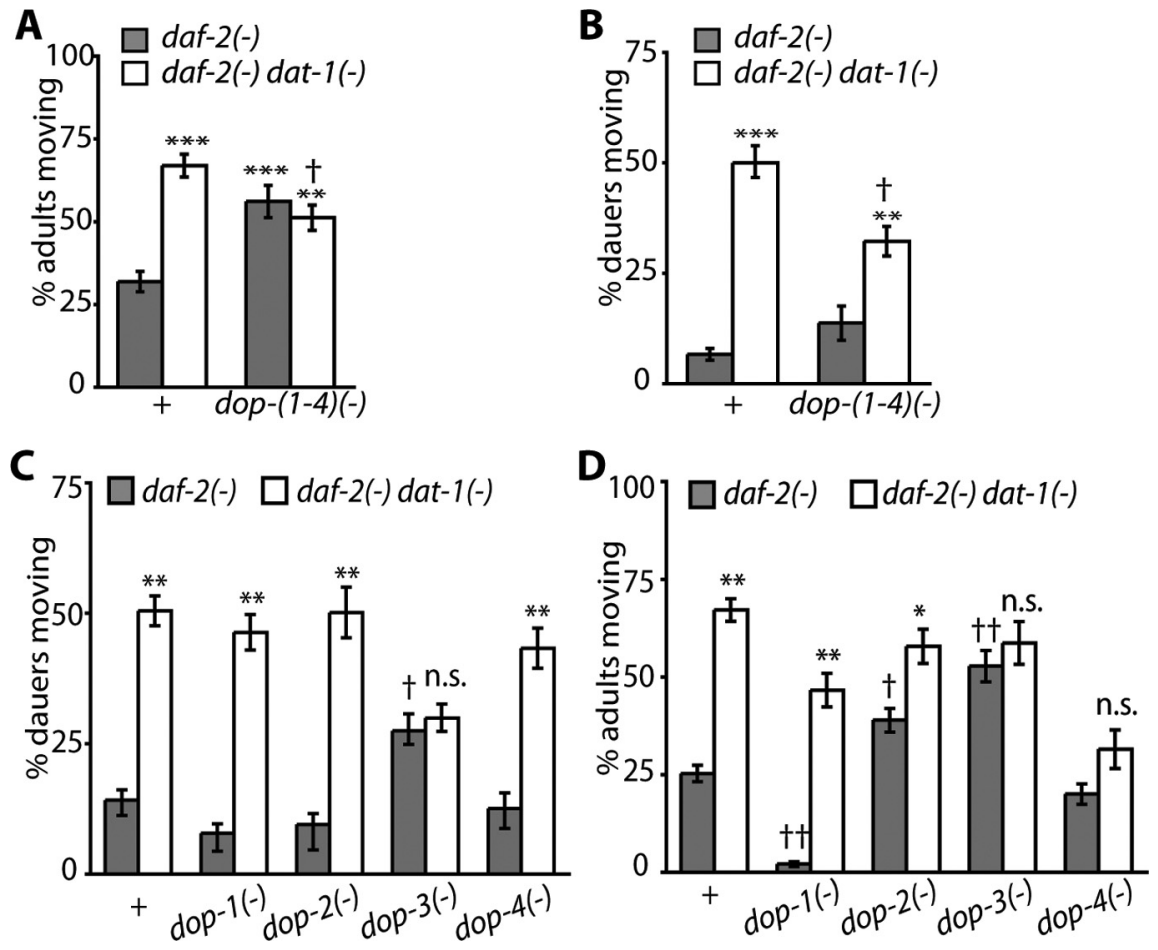

Figure 7. The DOP-3 receptor may be required for dat-1 mutations to increase daf-2/InsR mutant dauer and dauer-like adult motility. A, Mutating all four known dopamine receptors [dop- $(1-4)(-)]$ increased the movement of daf-2(-) adults. The effects of dat-1(-) mutation and of mutation of the four dopamine receptors on daf-2(-) adult quiescence were not additive. Percentage moving \pm SEM: daf-2(e1370), $32 \pm 3 \%$; daf-2(e1370); dop-2(vs105); dop-4(ok1321) dop-1(vs100) dop-3(vs106), $56 \pm 3 \%$; daf-2(e1370) dat-1(ok157), $67 \pm 5 \%$; daf-2(e1370) dat-1(ok157); dop-2(vs 105); dop-4(ok1321) dop-1(vs100) dop-3(vs106), $51 \pm 4 \% .{ }^{* * *} p<0.0001$ and ${ }^{* *} p<0.01$ versus daf-2(-) strain; ${ }^{\dagger} p<0.05$ versus daf-2(-) dat-1(-), Dunn-Sidak's corrected $t$ test. daf-2(-); dop- $(1-4)(-)$ and daf-2(-) dat-1(-); dop- $(1-$ $4)(-)$ were not significantly different from each other $(p=0.376)$. $\boldsymbol{B}$, Mutating all the four known C. elegans dopamine receptors $[$ dop- $(1-4)(-)]$ partially prevented the increase in motility of daf-2(-) dat-1(-) dauers. Percentage moving \pm SEM: daf-2(-), $7 \pm$ 1\%; daf-2(e1370); dop-2(vs105); dop-4(ok1321) dop-1(vs100) dop-3(vs106), $14 \pm 4 \%$; daf-2(e1370) dat-1(ok157), $50 \pm$ 4\%; daf-2(e1370) dat-1(ok157); dop-2(vs105); dop-4(ok1321) dop-1(vs100) dop3 (vs 106), $32 \pm 3 \%$. ${ }^{* *} p<0.0001$ and ${ }^{* *} p<0.01$ for each daf-2(-) dat-1(-) strain versus the corresponding daf-2(-) strain. ${ }^{\dagger} p<0.05$ versus daf-2(-) dat-1(-), Dunn-Sidak's corrected $t$ test. $C$, Loss of the D0P-3 receptor prevented dat- 1 mutations from increasing the motility of daf-2/InsR mutant dauers. Percentage moving \pm SEM: daf2(e1370), $14 \pm$ 2\%; daf-2(e1370) dat-1(ok157), $50 \pm 3 \%$; daf-2(e1370); dop-1(vs100), $8 \pm 2 \%$; daf-2(e1370) dat1(ok157); dop-1(vs100), $46 \pm 3 \%$; daf-2(e1370); dop-2(vs105), $10 \pm$ 2\%; daf-2(e1370) dat-1(ok157); dop-2(vs105), $50 \pm 5 \%$; daf-2(e1370); dop-3(vs106), $27 \pm 3 \%$; daf-2(e1370) dat-1(ok157); dop-3(vs106), $30 \pm 3 \%$; daf-2(e1370); dop-4(ok1321), $13 \pm 3 \%$; daf-2(e1370) dat-1(ok157); dop-4(ok1321), $43 \pm 4 \%$. ${ }^{* *} p<0.01$ and ${ }^{\text {n.s. } p}>0.05$ for each daf-2(-) dat-1(-) strain versus the corresponding daf-2(-) strain; ${ }^{\dagger} p<0.05$ versus daf-2(-), Dunn-Sidak's corrected $t$ test. $D$, Loss of the receptors DOP-3 and DOP-4 prevented dat-1 mutations from increasing the motility of daf-2/InsR mutant adults, whereas dop-1 mutations decreased the motility of both daf-2(-) and daf-2(-) dat-1(-) adults. Percentage moving \pm SEM: daf-2(e1370), $25 \pm 2 \%$; daf-2(e1370) dat-1(ok157), $67 \pm 3 \%$; daf-2(e1370); dop1(vs100), $2 \pm 1 \%$; daf-2(e1370) dat-1(ok157); dop-1(vs100), $47 \pm$ 4\%; daf-2(e1370); dop-2(vs105), $39 \pm 3 \%$; daf2(e1370) dat-1(ok157); dop-2(vs105), $58 \pm$ 4\%; daf-2(e1370); dop-3(vs106), $53 \pm$ 4\%; daf-2(e1370) dat-1(ok157); dop-3(vs106), $59 \pm$ 5\%; daf-2(e1370); dop-4(ok1321), $20 \pm 3 \%$; daf-2(e1370) dat-1(ok157); dop-4(ok1321), $31 \pm 5 \%$. ${ }^{* *} p<0.01,{ }^{*} p<0.05$, and ${ }^{n . s} . p>0.05$ for each daf-2(-) dat-1(-) strain versus the corresponding daf-2(-) strain. ${ }^{+\dagger} p<0.01$ and ${ }^{\dagger} p<0.05$ versus daf-2(-), Dunn-Sidak's corrected $t$ test. All adult animals were grown at $15^{\circ} \mathrm{C}$, shifted to $25^{\circ} \mathrm{C}$ at the $\mathrm{L} 4$ stage, and tested at day 3 of adulthood. Error bars represent SEM.

dopamine signaling, increase the percentage of daf-2/InsR mutant dauers and adults that move. Changes in dopamine signaling affect the animals' ability to sustain an increase in motion after stimulation, rather than their unstimulated, baseline quiescence. This finding is significant because it suggests that dopamine specifically affects the response of these animals to environmental cues. In addition, it indicates that changes in dopamine do not affect the movement of daf-2(-) animals by somehow suppressing the primary daf-2-(-) defect through upregulation of insulin/IGF-1 signaling.

We also found that daf-16/FOXO, a transcription factor that mediates most of the effects of daf-2/InsR mutations, controls the unstimulated baseline movement in daf-2/InsR mutant animals. Therefore, mutations in daf-16/ FOXO and dopamine signaling affect daf2/InsR mutant quiescence in different ways.

\section{Dauer-like quiescence is probably not caused by changes in dopamine signaling}

If the quiescence of dauers and that of dauer-like adults were directly caused by a change in dopamine signaling within the animal, it should be possible to produce dauer-like quiescence simply by altering dopaminergic pathways. However, neither the addition of exogenous dopamine, the dat-1 mutation, loss of dopamine-producing neurons, nor mutation of any dopamine receptors produced a quiescent dauer-like phenotype. Although quiescence could conceivably be caused by a more subtle change in dopamine signaling, it seems most likely that dauer-like quiescence is caused by changes in nondopaminergic pathways. Also, our results suggest that perhaps different neuronal populations are required for the distinct regulation of baseline and stimulated movement in quiescent daf-2/InsR mutant dauers and adults, because the dopaminergic system appears to have preferential effects on movement in response to stimulation.

\section{The basal slowing response is intact in daf-2/InsR mutants}

The loss of dopamine-producing neurons increases the movement of dauerlike animals, and we attribute this effect to mimicry of a food-deprived state. Dopamine is required for worms to slow down when they encounter food, and as a consequence, the increased locomotion of animals that lack dopamine consistently resembles that of animals deprived of food (Sawin et al., 2000; Chase et al., 2004; Li et al., 2006). The slowing response to food, called the basal slowing response, is thought to ensure that animals stay on food when they encounter it. We found that daf-2/InsR mutant animals display a basal slowing response, as their average speed is higher off food than on food. (However, food withdrawal is not sufficient to prevent quiescence, as daf-2/InsR mutant animals eventually become quiescent whether they are on food or not.) In addition, we found that mutations in the dop-3-receptor and dop-1-receptor genes affect daf-2/InsR mutant quiescence in a similar manner to the way they affect the basal slowing response in wild-type nondauer animals (Chase et al., 2004). We conclude that, in daf-2/InsR mutant animals, the dopamine circuit that controls the basal slowing response is mostly intact. 


\section{dat-1 mutations affect the quiescence of daf-2/InsR mutants through a novel mechanism}

Our results suggest that dat-1 mutations increase the locomotion of dauer-like daf-2/InsR mutant animals by a different mechanism, one that involves higher extracellular dopamine levels. Because dat-1 mutant animals can modulate their locomotion in response to food similarly to wild-type animals (supplemental Fig. S2 A, B, available at www.jneurosci.org as supplemental material), it is unlikely that the increase in extracellular dopamine simply leads to a compensatory decrease in dopamine signaling that mimics the basal slowing response (Jones et al., 1998). In addition, other phenotypes displayed by dat-1 mutants are consistent with their having elevated levels of dopamine signaling, such as slowed habituation on food (Kindt et al., 2007) and swimming-induced paralysis (McDonald et al., 2007). Therefore, we conclude that dat-1 mutations increase movement in daf-2/ InsR mutants by a mechanism that is novel and unrelated to the basal slowing response.

Because wild-type worms move more frequently and faster than daf-2/InsR animals, one possibility is that an increase in movement may be easier to detect in the quiescent daf-2(-) animals. However, it is possible to increase the speed of locomotion in wild type: for example, by food withdrawal, ablation of dopamine-producing neurons, and mutations in $d o p$-3 (Chase et al., 2004). Therefore, we reasoned that a stimulation of movement by dopamine should be detectable. We hypothesize that daf-2/InsR mutant dauers and adults are programmed to respond differently from nondauers to increases in dopamine signaling. The dopamine receptor DOP-3 appears to mediate the stimulating effect of increased dopamine signaling in daf-2/InsR mutant animals, and another receptor, DOP-4, may also be important, although only in adult animals.

In mammals, dopamine has been studied intensively both for its control of voluntary movement and for its role in reward pathways that underlie, among other things, drug-seeking behavior. It is possible that considering dopamine to be a reward signal in the context of dauer movement might explain how opposite interventions, decreasing and increasing dopamine, both result in similar behavioral outcomes. Also, it may explain why, whereas in C. elegans dopamine is generally thought to decrease movement (Schafer and Kenyon, 1995), in most other organisms dopamine is known to increase movement (Giros et al., 1996; Kume et al., 2005). Increases in dopamine when the worm encounters food (i.e., during the basal slowing response) may serve as a "reward" stimulus, encouraging the worm to consume the food rather than to keep moving. In dauers, however, where energy conservation and quiescence are the basal state, an additional system of rewards may be necessary to stimulate the worms to move in the first place, and thus dopamine release may be activated by particularly strong stimuli. For these two systems to coexist, the dopamine signaling system would need to be altered in dauer and dauer-like animals so as to make the animals more sensitive to the stimulatory effects of dopamine. Our data suggest that this alteration may be attributable to changes in the activity of the C. elegans daf-2/InsR pathway.

Our findings indicate that the DAF-16/FOXO transcription factor, which is known to be activated by reduction of insulin/ IGF-1 signaling, is required for daf-2 mutants to move faster in response to increased dopamine signaling (i.e., dat-1 mutations). We observed that, in daf-16(-); daf-2(-) double mutants, which move more like wild type, dat-1 mutations increase the percentage of animals moving, but have only a small effect on their speed. This resembles the situation in wild-type animals. In addition, restoring daf-16/FOXO in the neurons is sufficient for daf-16(-); daf-2(-) double mutants to become quiescent. Thus, transcriptional changes controlled by DAF-16/FOXO in neurons may underlie the different responses to dopamine displayed by daf- $2 /$ InsR mutants. DAF-16/FOXO and DAF-2/InsR are both expressed widely in the nervous system and thus could affect dopamine signaling by changing gene expression in cells that release or respond to dopamine. However, the insulin/IGF-1 signaling pathway could also regulate the dopamine pathway in a cell-nonautonomous manner, as it does in its regulation of life span and dauer formation (Apfeld and Kenyon, 1998; Wolkow et al., 2000; Libina et al., 2003).

We tested whether any of the known components of the dopamine pathway are targets of DAF-16/FOXO. Two genes were downregulated in daf-2/InsR mutant animals compared with wild-type animals: bas-1, an aromatic amino acid decarboxylase required for synthesis of dopamine and serotonin, and dop-2, one of the dopamine receptors (data not shown) [also reported in McElwee et al. (2004)]. However, these changes do not explain our findings, as there was no detectable difference in the overall levels of dopamine in wild-type versus daf-2(e1370) animals (supplemental Fig. S7, available at www.jneurosci.org as supplemental material) (tested as day 3 adults by HPLC analysis), although the data do not rule out more subtle changes in distribution of dopamine in the animal. In addition, daf-2(-) dat-1(-); dop-2(-) animals still moved faster than daf-2(-); dop-2(-) animals (Fig. $7 C, D$ ). It would be interesting to test all candidate DAF-16-regulated genes for their effects on quiescence, but because RNA interference does not work well in neurons (Timmons et al., 2001), this is not a simple task. Perhaps critical genes could be recovered in a screen for mutations that stimulate the movement of quiescent daf-2(-) animals.

\section{Insulin regulation of dopamine signaling in other organisms}

The idea that the insulin/IGF-1 signaling pathway can alter the activity of the dopamine pathway in C. elegans is compatible with the emerging idea that, in the mammalian brain, hormonal signals involved in energy balance, including insulin, can affect the dopamine reward pathway to modulate feeding behavior ( $\mathrm{Fi}$ glewicz et al., 2007; Palmiter, 2007). Food-restricted animals, which have lower circulating insulin levels, behave as though they have elevated dopamine signaling. However, the mechanism by which insulin signaling acts at the cellular level to influence dopamine signaling is still unclear.

In the mammalian brain, some of the effects of dopamine are not attributable to simple increase or decrease of dopamine, but rather to changes in the kinetics of dopamine release (Goto et al., 2007). In C. elegans, many of the subtleties of dopamine signaling kinetics that are important for dopamine signaling in other organisms (Goto et al., 2007) are still unexplored, because all studies, including this one, have relied on generalized loss of dopamine production or unspecific increases in dopamine levels. Therefore, the study of daf-2/InsR mutant movement in response to stimulation may be an interesting paradigm in which to explore the worm's dopamine system in more detail.

\section{Implications for hibernation}

Quiescent C. elegans dauers resemble animals in hibernation in many ways (Carey et al., 2003), although, unlike in C. elegans, hibernation in higher animals does not occur only at a specific prepubescent developmental stage. Interestingly, neurons of hibernating mammals have decreased activity of Akt (Lee et al., 2002; Eddy and Storey, 2003; Cai et al., 2004; Abnous et al., 2008), 
which is one of the kinases that inhibits DAF-16/FOXO under replete conditions. Thus, hibernation and the dauer diapause may be similar at a molecular level. It is even possible that hibernation is potentiated, in part, by a reduction in insulin/IGF-1 signaling caused by nutrient deprivation or other stressful conditions. During hibernation, suppression of movement is important to prevent energy expenditure, but little is known about how suppression of movement is regulated in this context. It would be interesting to know whether hibernating animals are particularly susceptible to the stimulatory effects of dopamine. Likewise, in $C$. elegans, it would be interesting to know whether there are environmental stimulants that activate quiescent dauers by elevating dopamine signaling.

It seems possible that the ability to suppress movement is an ancient response to prolonged environmental stress that is expressed in the context of the dauer in C. elegans and in the context of hibernation (and possibly sleep) in other animals. If so, then understanding the neural pathways that regulate dauer behavior, which are genetically accessible, may have more general implications for the behavior of higher organisms.

\section{References}

Abnous K, Dieni CA, Storey KB (2008) Regulation of Akt during hibernation in Richardson's ground squirrels. Biochim Biophys Acta 1780:185-193.

Apfeld J, Kenyon C (1998) Cell nonautonomy of C. elegans daf-2 function in the regulation of diapause and life span. Cell 95:199-210.

Birnby DA, Link EM, Vowels JJ, Tian H, Colacurcio PL, Thomas JH (2000) A transmembrane guanylyl cyclase (DAF-11) and Hsp90 (DAF-21) regulate a common set of chemosensory behaviors in Caenorhabditis elegans. Genetics 155:85-104.

Brenner S (1974) The genetics of Caenorhabditis elegans. Genetics 77:71-94. Brockie PJ, Madsen DM, Zheng Y, Mellem J, Maricq AV (2001) Differential expression of glutamate receptor subunits in the nervous system of Caenorhabditis elegans and their regulation by the homeodomain protein UNC-42. J Neurosci 21:1510-1522.

Cai D, McCarron RM, Yu EZ, Li Y, Hallenbeck J (2004) Akt phosphorylation and kinase activity are down-regulated during hibernation in the 13-lined ground squirrel. Brain Res 1014:14-21.

Carey HV, Andrews MT, Martin SL (2003) Mammalian hibernation: cellular and molecular responses to depressed metabolism and low temperature. Physiol Rev 83:1153-1181.

Cassada RC, Russell RL (1975) The dauerlarva, a post-embryonic developmental variant of the nematode Caenorhabditis elegans. Dev Biol 46:326-342.

Chase DL, Pepper JS, Koelle MR (2004) Mechanism of extrasynaptic dopamine signaling in Caenorhabditis elegans. Nat Neurosci 7:1096-1103.

Eddy SF, Storey KB (2003) Differential expression of Akt, PPARgamma, and PGC-1 during hibernation in bats. Biochem Cell Biol 81:269-274.

Figlewicz DP, MacDonald Naleid A, Sipols AJ (2007) Modulation of food reward by adiposity signals. Physiol Behav 91:473-478.

Gainetdinov RR, Caron MG (2003) Monoamine transporters: from genes to behavior. Annu Rev Pharmacol Toxicol 43:261-284.

Gems D, Sutton AJ, Sundermeyer ML, Albert PS, King KV, Edgley ML, Larsen PL, Riddle DL (1998) Two pleiotropic classes of daf-2 mutation affect larval arrest, adult behavior, reproduction and longevity in Caenorhabditis elegans. Genetics 150:129-155.

Giros B, Jaber M, Jones SR, Wightman RM, Caron MG (1996) Hyperlocomotion and indifference to cocaine and amphetamine in mice lacking the dopamine transporter. Nature 379:606-612.

Goto Y, Otani S, Grace AA (2007) The Yin and Yang of dopamine release: a new perspective. Neuropharmacology 53:583-587.

Gottlieb S, Ruvkun G (1994) daf-2, daf-16 and daf-23: genetically interacting genes controlling Dauer formation in Caenorhabditis elegans. Genetics 137:107-120.

Haycraft CJ, Swoboda P, Taulman PD, Thomas JH, Yoder BK (2001) The C. elegans homolog of the murine cystic kidney disease gene Tg737 functions in a ciliogenic pathway and is disrupted in osm-5 mutant worms. Development 128:1493-1505.
Henderson ST, Johnson TE (2001) daf-16 integrates developmental and environmental inputs to mediate aging in the nematode Caenorhabditis elegans. Curr Biol 11:1975-1980.

Hills T, Brockie PJ, Maricq AV (2004) Dopamine and glutamate control area-restricted search behavior in Caenorhabditis elegans. J Neurosci 24:1217-1225.

Hu PJ (2007) Dauer. WormBook 1-19.

Jayanthi LD, Apparsundaram S, Malone MD, Ward E, Miller DM, Eppler M, Blakely RD (1998) The Caenorhabditis elegans gene T23G5.5 encodes an antidepressant- and cocaine-sensitive dopamine transporter. Mol Pharmacol 54:601-609.

Jones SR, Gainetdinov RR, Jaber M, Giros B, Wightman RM, Caron MG (1998) Profound neuronal plasticity in response to inactivation of the dopamine transporter. Proc Natl Acad Sci U S A 95:4029-4034.

Kimura KD, Tissenbaum HA, Liu Y, Ruvkun G (1997) daf-2, an insulin receptor-like gene that regulates longevity and diapause in Caenorhabditis elegans. Science 277:942-946.

Kindt KS, Quast KB, Giles AC, De S, Hendrey D, Nicastro I, Rankin CH, Schafer WR (2007) Dopamine mediates context-dependent modulation of sensory plasticity in C. elegans. Neuron 55:662-676.

Kume K, Kume S, Park SK, Hirsh J, Jackson FR (2005) Dopamine is a regulator of arousal in the fruit fly. J Neurosci 25:7377-7384.

Lee BH, Ashrafi K (2008) A TRPV channel modulates C. elegans neurosecretion, larval starvation survival, and adult lifespan. PLoS Genet 4:e1000213.

Lee M, Choi I, Park K (2002) Activation of stress signaling molecules in bat brain during arousal from hibernation. J Neurochem 82:867-873.

Lee RY, Hench J, Ruvkun G (2001) Regulation of C. elegans DAF-16 and its human ortholog FKHRL by the daf-2 insulin-like signaling pathway. Curr Biol 11:1950-1957.

Li W, Feng Z, Sternberg PW, Xu XZ (2006) A C. elegans stretch receptor neuron revealed by a mechanosensitive TRP channel homologue. Nature 440:684-687.

Libina N, Berman JR, Kenyon C (2003) Tissue-specific activities of C. elegans DAF-16 in the regulation of lifespan. Cell 115:489-502.

Lin K, Dorman JB, Rodan A, Kenyon C (1997) daf-16: an HNF-3/forkhead family member that can function to double the life-span of Caenorhabditis elegans. Science 278:1319-1322.

Lin K, Hsin H, Libina N, Kenyon C (2001) Regulation of the Caenorhabditis elegans longevity protein DAF-16 by insulin/IGF-1 and germline signaling. Nat Genet 28:139-145.

McDonald PW, Hardie SL, Jessen TN, Carvelli L, Matthies DS, Blakely RD (2007) Vigorous motor activity in Caenorhabditis elegans requires efficient clearance of dopamine mediated by synaptic localization of the dopamine transporter DAT-1. J Neurosci 27:14216-14227.

McElwee JJ, Schuster E, Blanc E, Thomas JH, Gems D (2004) Shared transcriptional signature in Caenorhabditis elegans Dauer larvae and longlived daf-2 mutants implicates detoxification system in longevity assurance. J Biol Chem 279:44533-44543.

Mello C, Fire A (1995) DNA transformation. Methods Cell Biol 48:451-482.

Nass R, Hall DH, Miller DM III, Blakely RD (2002) Neurotoxin-induced degeneration of dopamine neurons in Caenorhabditis elegans. Proc Natl Acad Sci U S A 99:3264-3269.

Ogg S, Paradis S, Gottlieb S, Patterson GI, Lee L, Tissenbaum HA, Ruvkun G (1997) The Fork head transcription factor DAF-16 transduces insulinlike metabolic and longevity signals in C. elegans. Nature 389:994-999.

Palmiter RD (2007) Is dopamine a physiologically relevant mediator of feeding behavior? Trends Neurosci 30:375-381.

Ramot D, Johnson BE, Berry TL Jr, Carnell L, Goodman MB (2008) The Parallel Worm Tracker: a platform for measuring average speed and druginduced paralysis in nematodes. PLoS ONE 3:e2208.

Ren P, Lim CS, Johnsen R, Albert PS, Pilgrim D, Riddle DL (1996) Control of C. elegans larval development by neuronal expression of a TGF-beta homolog. Science 274:1389-1391.

Riddle DL, Albert PS (1997) Regulation of dauer larva development. In: C. elegans II (Riddle DL, Blumenthal T, Meyer BJ, Priess JR, eds), pp 739768. Cold Spring Harbor, NY: Cold Spring Harbor Laboratory.

Riddle DL, Swanson MM, Albert PS (1981) Interacting genes in nematode dauer larva formation. Nature 290:668-671.

Sawin ER, Ranganathan R, Horvitz HR (2000) C. elegans locomotory rate is 
modulated by the environment through a dopaminergic pathway and by experience through a serotonergic pathway. Neuron 26:619-631.

Schafer WR, Kenyon CJ (1995) A calcium-channel homologue required for adaptation to dopamine and serotonin in Caenorhabditis elegans. Nature 375:73-78.

Sugiura M, Fuke S, Suo S, Sasagawa N, Van Tol HH, Ishiura S (2005) Characterization of a novel D2-like dopamine receptor with a truncated splice variant and a D1-like dopamine receptor unique to invertebrates from Caenorhabditis elegans. J Neurochem 94:1146-1157.

Sulston J, Dew M, Brenner S (1975) Dopaminergic neurons in the nematode Caenorhabditis elegans. J Comp Neurol 163:215-226.

Suo S, Sasagawa N, Ishiura S (2002) Identification of a dopamine receptor from Caenorhabditis elegans. Neurosci Lett 319:13-16.

Suo S, Sasagawa N, Ishiura S (2003) Cloning and characterization of a Cae- norhabditis elegans D2-like dopamine receptor. J Neurochem 86:869-878.

Thomas JH, Birnby DA, Vowels JJ (1993) Evidence for parallel processing of sensory information controlling dauer formation in Caenorhabditis elegans. Genetics 134:1105-1117.

Timmons L, Court DL, Fire A (2001) Ingestion of bacterially expressed dsRNAs can produce specific and potent genetic interference in Caenorhabditis elegans. Gene 263:103-112.

Vowels JJ, Thomas JH (1992) Genetic analysis of chemosensory control of dauer formation in Caenorhabditis elegans. Genetics 130:105-123.

Wolkow CA, Kimura KD, Lee MS, Ruvkun G (2000) Regulation of C. elegans life-span by insulinlike signaling in the nervous system. Science 290:147-150. 